\title{
Reasoned Modelling with Event-B
}

\author{
Michael Butler \\ 30 January 2017 \\ University of Southampton
}

\begin{abstract}
This paper provides an overview of how the Event-B language and verification method can be used to model and reason about system behaviour. Formal modelling and reasoning help to increase understanding and reduce defects in requirements specification. Sets and relations play a key role in modelling as do operators on these structures. Precise definitions and rules are provided in order to help the reader gain a strong understanding of the mathematical operators for sets and relations. While the emphasis is on mathematical reasoning, particularly through invariant proofs, the paper also covers less formal reasoning such as identification of problem entities supported by class diagrams and validation of formal models against informal requirements. The use of tools for animation, model checking and proof is also outlined.
\end{abstract}

\section{Introduction}

This paper provides an introduction to formal modelling using the Event-B language and method [1]. We make no strong assumptions about the existing knowledge of the reader other than in interest in learning about the approach and a willingness to start to put it into practice.

It is useful to motivate the role and value of the formal methods that we are outlining and advocating in this paper. Essentially it is about improving the processes that are used to engineer software-based systems so that specification and design errors are identified and rectified as soon as possible in the system development cycle. From the earliest days of software engineering it has been recognised that the cost of fixing a specification or design error is higher the later in the development that error is identified. This is summarised by the following observation about software development by Boehm [2]:

Boehm's First Law: Errors are more frequent during requirements and design activities and are more expensive the later they are removed.

This observation is bourne out by many studies of software engineering projects. For example, a 2013 report from the Carnegie-Mellon Software Engineering Institute (SEI) highlights studies showing that requirements and architecture defects make up approximately $70 \%$ of all system defects and that $80 \%$ of these defects are discovered late in the development life cycle [3]. 


\section{Early identification of errors through formal modelling}

Clearly, identifying errors at the point at which they have become expensive to fix, long after they were introduced, is undesirable. More desirable would be to discover errors as soon as possible when they are less expensive to fix. So, why is it difficult to achieve this ideal profile in practice? Common errors introduced in the early stages of development are errors in understanding the system requirements and errors in writing the system specification. Without a rigorous approach to understanding requirements and constructing specifications, it can be very difficult to uncover such errors other than through testing of the software product after a lot of development has already been undertaken. Why is it difficult to identify errors that are introduced early in the development cycle? One reason is lack of precision in formulating specifications resulting in ambiguities and inconsistencies that are difficult to detect and may store up problems for later. Another reason is too much complexity, whether it is complexity of requirements, complexity of the operating environment of a system or complexity of the design of a system.

To overcome the problem of lack of precision, we advocate the the use of formal modelling. As well as encouraging precise descriptions, formal modelling languages are supported by verification methods that support the discovery and elimination of inconsistencies in models. But precision on its own does not address the problem of complex requirements and operating environments. Complexity cannot be eliminated but we can try to master it. To master complexity, we advocate the use of abstraction. Abstraction is about simplifying our understanding of a system to arrive at a model that is focused on what we judge to be the key or critical features of a system. A good abstraction will focus on the purpose of a system and will ignore details of how that purpose is achieved. We do not ignore the complexity indefinitely: instead, through incremental modelling and analysis, we can layer our understanding and analysis of a system. This incremental treatment of complexity is the other side of the coin to abstraction, namely, refinement.

The Event-B modelling approach is intended for early stage analysis of computer systems. It provides a rich modelling language, based on set theory, that allows precise descriptions of intended system behaviour (models) to be written in an abstract way. It provides a mathematical notion of consistency together with techniques for identifying inconsistencies or verifying consistency within a model. It also provides a notion of refinement of models together with a notion of consistency between a model and its refinement. By abstracting and modelling system behaviour in Event-B, it is possible to identify and fix requirements ambiguities and inconsistencies at the specification phase, much earlier in the development cycle than system testing. In this way, rather than having an error-discovery profile in which most errors are discovered during system testing, we would arrive at an ideal profile in which more errors are discovered as soon as they are introduced. This paper will focus on precision and verification of consistencies in abstract specifications and does not cover refinement of models. Section 13 points to some further reading on refinement. 


\section{Requirements and formal models}

We assume that the results of any requirements analysis phase is a requirements document written in natural language. There remains a potentially large gap between these informal requirements and a formal model. In this paper we will touch on this gap but not address it in any comprehensive way. In the context of a system development that involves both informal requirements and formal specification, it is useful to distinguish two notions of validation as follows:

- Requirements validation involves analysing the extent to which the (informal) requirements satisfy the needs of the stakeholders.

- Model validation involves analysing the extent to which the (formal) model accurately captures the (informal) requirements.

Both of these forms of validation require the use of human judgement, ideally by a range of stakeholders. In addition, we can perform mathematical judgements on a formal model. We refer to this use of mathematical judgements are model verification, that is, the extent to which a model satisfies a given set of mathematical judgements. Key to the effective use of model verification is strong tool support that automates the verification effort as much as possible. Arriving at good abstractions, formalising them, enriching models through refinement and making mathematical judgements all require skill and effort. This upfront effort is sometimes referred to as front-loading: putting more effort than is usual into the early development stages in order to save test and fix effort later.

\section{Overview of paper}

Logic and set theory are the mathematical basis of Event-B. In this paper we explain how these mathematical concepts are used to write precise specifications in the form of Event-B models and how we reason about such models using mathematics. We use sets as a form of abstract data structure to model collections of entities that have a certain status and we define events that specify ways in which these sets may be manipulated to represent changes in the status of entities. For example, Section 2 shows how a set is used to model collections of users who have permission to be in a building and presents events for adding users to this set when they are registered and for removing users from this set when they are de-registered. Mathematical operators on sets allow us to easily specify manipulations of sets and Section 3 provides a brief overview of the set operators used throughout this paper while Section 5, covers issues that arise with finiteness of sets and determining the size of sets.

Sets are used to model collections of entities of the same kind. When we want to model connections between different kinds of entities, we use relations which are covered in Sections 6 and 7 .

The main unit of specification in Event-B is a machine and this is introduced in Section 4. A machine contains a list of variables and a list of events that modify the variables in precisely defined ways. A machine also contains a list of 
invariants that describe desired properties of the variables of a machine, e.g., users inside the building must have permission to be there.

Many set operators are defined using mathematical logic. For example, intersection of sets is defined in terms of logical conjunction: an element $x$ is in the intersection of sets $S$ and $T$ if $x$ is in $S$ and $x$ is in $T$. Section 3 gives a brief overview of the main logical operators used and the connection between logic and sets. At various stages in the paper additional mathematical operators are introduced to support the required modelling. Mathematical definitions are provided to help the reader's understanding of the operators and to support mathematical reasoning. Logic allows us to reason about machines, in particular, it allows us to prove that events of a machine preserve constraints specified by invariants and this is covered in several places in the paper.

We use several case studies to illustrate the use of the modelling and reasoning concepts of Event-B. Sections 2-4 use a simple example of a system for controlling access to a building. This case study is used to in illustrate the use of sets as abstract data structures and the use of invariants for specifying desired properties of structures. The case study is also used to provide the initial illustration of the use of mathematical reasoning to verify properties of a machine. In Sections 8 and 9 we use a generalisation of the access control system that manages access to a collection of buildings rather than a single building. This case study is used to illustrate the use of relations (e.g., between users and buildings) and to consolidate the concepts from the earlier sections. A function is a special case of a relation and we use an example of a simple banking system to illustrate the use of functions in modelling in Section 10.

While reading and understanding a specification written in a language such as Event-B requires a relatively small amount of training, the ability to write a formal specification requires more skill and, as with programming, that skill is best developed through practice. Using the access control example, Sections 8 and 9 provide guidelines on how an Event-B model can be constructed from a list of informal system requirements. The author has found that the use of class diagrams provides a useful initial bridge between informal requirements and formal models involving relations and functions. Class diagrams help to identify in a graphical way the various entities appropriate for a system model and the various connections between the different kinds of entities. For example, in an access control model, users and buildings are two relevant entities and the access rights are represented by an association between those entities.

A key advantage of Event-B is the availability of tool support for reasoning about formal models. Sections 11 and 12 provide an overview of tool support (animation, model checking, proof obligation generation and automated proof) that is available to support model validation and verification.

Section 13 briefly overviews some material that provides a deeper treatment of Event-B than this paper and also overviews other related formal modelling and analysis methods. 


\section{Modelling with sets and invariants}

We illustrate modelling with sets through an example of access control to a building. The system should allow only registered users to enter the building and should keep track of which users are inside the building. We start by considering two sets, in, representing the users who are inside the building and out, representing the users who are outside the building. The two sets are illustrated by the Venn diagram in Figure 1.

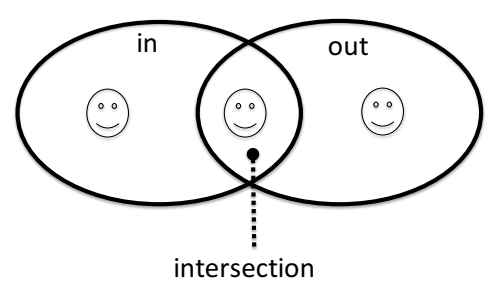

Fig. 1. Venn diagram for in and out

The diagram illustrates that we might have users in the overlapping area between the two sets (the intersection) and users in the non-overlapping areas. However, for this particular example, we would not expect any users to be both inside and outside the building so we would like to rule this possibility out as illustrated in Figure 2. When the intersection of two sets is empty, we say the

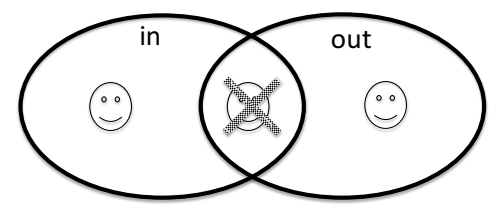

Fig. 2. Venn diagram: empty intersection

sets are disjoint and disjointness is illustrated in Figure 3 by having no overlap between the sets. This disjointness property may be represented by the following mathematical equation:

$$
\text { in } \cap \text { out }=\varnothing
$$

The equation says that the intersection of the two sets $(i n \cap$ out $)$ is empty $(\varnothing)$.

The system we are modelling is dynamic in that users may enter or leave the building. In our model, this will be reflected by changes to the sets in and out. Thus we treat in and out as variables whose values may be changed. We make 


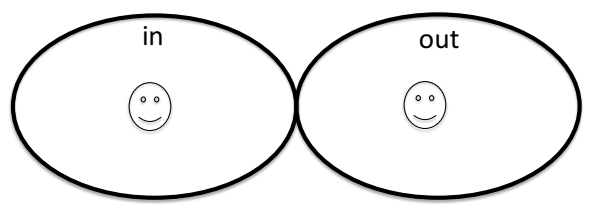

Fig. 3. Venn diagram: disjoint sets

the following declaration:

$$
\text { variables in, out }
$$

While the values of the variables may change, the disjointness property should remain true. An invariant is a property of one or more variables that should be preserved by an changes to the variables so, not matter what changes occur in the system, it should never get into a state in which the invariant is falsified. We require the disjointness equation to be an invariant of our access control model so we declare:

\section{invariant in $\cap$ out $=\varnothing$}

We mentioned the concept of registered users at the beginning of this section so we introduce a set, called register, representing registered users. We will allow the set of registered user to change, e.g., by adding a new user to the register, so we declare register to be a variable:

\section{variable register}

Only registered users should be allowed in the building and we model this property by requiring in to be contained entirely within register as illustrated in Figure 4. As the diagram illustrates, a user who is in the building must also be registered. The diagram also illustrates that some users may be registered without being in the building. We say that in is a subset of register, written in mathematical notation as: in $\subseteq$ register. We declare this subset property on in and register as an invariant:

$$
\text { invariant } \text { in } \subseteq \text { register }
$$

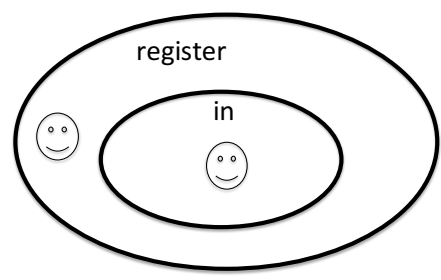

Fig. 4. Venn diagram: subset 
What about the relationship between the set out and the set register? Up to now we have not been clear about whether out represents all possible users including those that are not registered. Let us make a modelling decision that out represents exactly those users who are registered and are outside the building. Thus registered users are either in or out. This is illustrated by Figure 5 which shows that register is the union of in and out. Mathematically, the union is written as in $\cup$ out and we declare the union property as an invariant:

invariant register $=$ in $\cup$ out

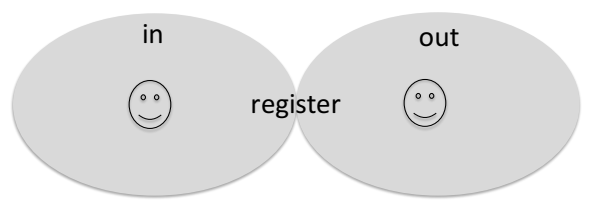

Fig. 5. Venn diagram: set union

We can add behaviours to the model, such as a user entering the building or leaving the building, by specifying events. An event defines an atomic transition on states, that is, it defines a relationship between a state before the event is executed and the resulting state after the event is executed. An atomic transition representing a user entering the building is illustrated by Figure 6 . This shows Venn diagrams for the variables in and out both before and after execution of the Enter event. In the before state, user $u$ is in the set out while in the after state $u$ is in the set $i n$, i.e., the Enter event moves user $u$ from out to in. As shown in Figure 6, the specification of the Enter event has three parts:

- parameter $u$ representing the user who is entering the building

- a guard requiring that the user is in the set out

- an action that moves $u$ from out to in,

The Enter event is specified in Event-B notation as follows (including comments):

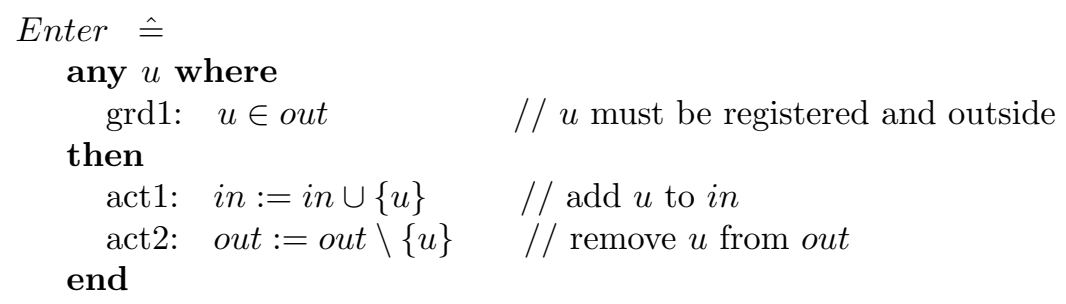

Here the keyword any indicates that $u$ is a parameter. The guard of the event appears between the where and then keywords while the actions appears 


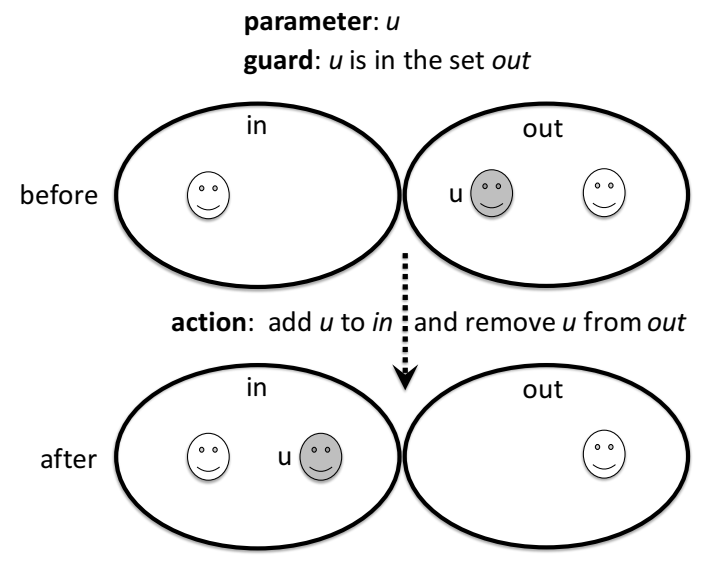

Fig. 6. Illustrating the Enter event

between the then and end keywords. The guard labelled $\operatorname{grd} 1$ requires that $u$ is in the set out, written $u \in$ out. The actions of the event specify assignments that modify some of the variables of the model, e.g., the action labelled act 1 assigns the value in $\cup\{u\}$ to the variable $i n$. The action labelled act2 uses set difference to remove $u$ from out: $s \backslash t$ is the difference between sets $s$ and $t$, i.e,. the set elements of $s$ that are not in $t$.

Although the Enter event contains several actions, the order in which the actions appear does not matter as all of the actions are executed together, not in series.

The syntax for specifying events will be described systematically in Section 4 . Before presenting further details of the model of the building access control we give a quick overview, in the next section, of the key concepts of set theory that are important in the Event-B notation.

\section{Overview of Set Theory}

Here we list some key features of sets:

- A set is a collection of elements.

- Elements are not ordered by a set.

- Set membership is an important relationship between an element and a set. We write $x \in S$ to specify that element $x$ is a member of set $S$.

- Elements may themselves be sets, i.e., we can have a set of sets.

- Sets may be enumerated within braces, e.g., the set $\{a, b, c\}$ contains three elements, $a, b$ and $c$.

- The set containing no elements, the empty set, is written $\varnothing$.

Set membership is a boolean property relating an element and a set, i.e., either $x$ is in $S$ or $x$ is not in $S$. This means that there is no concept of an 
element occurring more that once in a set, e.g.,

$$
\{a, a, b, c\}=\{a, b, c\} .
$$

Set membership says nothing about the relationship between the elements of a set other than that they are members of the same set. This means that the order in which we enumerate a set is not significant, e.g.,

$$
\{a, b, c\}=\{b, a, c\} .
$$

These two characteristics distinguish sets from data structures such as lists or arrays where elements appear in order and the same element my occur multiple times.

\subsection{Typing and powersets}

All the elements of a set must have the same type where a type is a special kind of set known as a carrier set. Figure 7 illustrates that the variables register, in and out are all subsets of the carrier set USER. In Event-B we use an invariant to define the carrier set of a variable, e.g.,

\section{invariant register $\subseteq U S E R$}

This declaration means that all the elements of the set register have the type $U S E R$. If we also have the invariant register $=i n \cup$ out, the elements of in and out must have the same type as the elements of register. That is, the type of in and out can be inferred from the type of register because of the invariant register $=$ in $\cup$ out. All the elements of a set must have the same type.

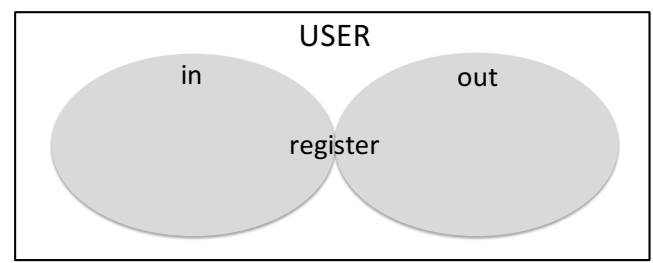

Fig. 7. Carrier set USER

A carrier set is maximal in that it is not a subset of any other set. A model may contain several carrier sets and these are implicitly disjoint from each other. For example, we could have a model that contains two carrier sets USER and $B U I L D I N G$. We cannot combine carrier sets using set union, intersection or difference, e.g., $U S E R \cup B U I L D I N G$ is invalid. In Section 6 we will see that we can combine carrier sets in another way to form relations. A carrier set remains fixed during the execution of a model, i.e., actions of an event cannot assign to a carrier set. 
Suppose $C$ is a carrier set. To define the type of an element $x$ to be $C$, we simply declare $x \in C$. If $S$ is not a carrier set and $S \subseteq C$, then the declaration $x \in S$ means that the type of $x$ is $C$.

The Event-B notation has an in-built carrier set representing integers, written $\mathbb{Z}$. Elements of this set can be written using the usual literals, e.g., 1, 2, 3. The Event-B notation supports the usual arithmetic operators for integers such as addition and multiplication.

A powerset of a set is the set of all subsets of that set. For set $S$, we write $\mathbb{P}(S)$ for the powerset of $S$. For example,

$$
\mathbb{P}(\{a, b, c\})=\{\varnothing,\{a\},\{b\},\{c\},\{a, b\},\{a, c\},\{b, c\},\{a, b, c\}\}
$$

Note that both the empty set and the set itself are contained within a set's powerset.

Up to now we have referred to the type of the elements of a set, e.g., all the elements of register have type USER. What about the type of the set itself (as opposed to the elements of the set)? We use the powerset operator to define the type of the set itself:

If the elements of a set $S$ are of type $C$ then the type of $S$ is $\mathbb{P}(C)$.

For example, we have:

- the type of the set register is $\mathbb{P}(U S E R)$

- the type of the set $\{1,2,3\}$ is $\mathbb{P}(\mathbb{Z})$

\subsection{Expressions and predicates}

Expressions are syntactic structures for specifying values (elements or sets). Literals (e.g., 3, $\varnothing$ ) are basic expressions as are variables (e.g., register) and carrier sets (e.g., USER). Compound expressions are formed by applying expressions to operators such as $x+y$ and $S \cup T$ to any level of nesting.

Predicates are syntactic structures for specifying logical statements, i.e., statements that are either true or false (but not both). Equality of expressions is an example predicate, e.g., register $=i n \cup$ out. Set membership and subset relations are other examples. For integer elements we can write ordering predicates such as $x \leq y$. Assume that $a, S, T, x$ and $y$ are expressions $(S$ and $T$ are set expressions while $x$ and $y$ are integer expressions). We have available the following basic predicates:

Basic predicates: $\quad a \in S, \quad S \subseteq T, \quad S=T, \quad x=y, \quad x<y, \quad x \leq y$

Predicate operators: Compound predicates are formed using the standard logical operators listed in the following table (assume $P$ and $Q$ are predicates): 


\begin{tabular}{|l|l|l|}
\hline Name & Predicate & Definition \\
\hline Negation & $\neg P$ & $P$ does not hold \\
\hline Conjunction & $P \wedge Q$ & both $P$ and $Q$ hold \\
\hline Disjunction & $P \vee Q$ & either $P$ holds or $Q$ holds \\
\hline Implication & $P \Rightarrow Q$ & if $P$ holds, then $Q$ holds \\
\hline
\end{tabular}

Quantified predicates: We have seen that a predicate $P$ may refer to one or more variables, e.g., $x \leq y$. We can quantify over a variable of a predicate universally or existentially:

\begin{tabular}{|l|l|l|}
\hline Name & Predicate & Definition \\
\hline Universal Quantification & $\forall x \cdot P$ & $P$ holds for all $x$ \\
\hline Existential Quantification & $\exists x \cdot P$ & $P$ holds for some $x$ \\
\hline
\end{tabular}

In the predicate $\forall x \cdot P$ the quantification is over all possible values in the type of the variable $x$. Typically we constrain the range of values using implication, e.g., we could specify that every element of the set in is also an element of the set register:

$$
\forall u \cdot u \in i n \Rightarrow u \in \text { register }
$$

In the case of existential quantification we typically constraint the range of values using conjunction, e.g., we could specify that integer $z$ has a positive square root as follows:

$$
\exists y \cdot 0 \leq y \wedge y \times y=z
$$

Free and bound variables: A variable that is universally or existentially quantified in a predicate is said to be a bound variable. A variable referenced in a predicate that is not bound variable is called a free variable. For example, in the above predicate, $y$ is bound while $z$ is free.

Predicates on sets can be defined in terms of the logical operators as follows: 


\begin{tabular}{|l|l|l|}
\hline Name & Predicate & Definition \\
\hline Subset & $S \subseteq T$ & $\forall x \cdot x \in S \Rightarrow x \in T$ \\
\hline Set equality & $S=T$ & $S \subseteq T \wedge T \subseteq S$ \\
\hline
\end{tabular}

\subsection{Set Operators}

We already used expression operators on sets such as union and intersection. We now defines these operators more precisely using predicates. A predicate provides a way of defining a set: the set of elements that satisfy the predicate. Consider the union $S \cup T$. The elements of the union are those elements that are either in $S$ or in $T$. More precisely, the set $S \cup T$ is defined by the set of elements $x$ satisfying the predicate $x \in S \vee x \in T$. The following table provides definitions of the set operators using logical operators:

\begin{tabular}{|l|l|l|}
\hline Name & Predicate & Definition \\
\hline Union & $x \in S \cup T$ & $x \in S \vee x \in T$ \\
\hline Intersection & $x \in S \cap T$ & $x \in S \wedge x \in T$ \\
\hline Difference & $x \in S \backslash T$ & $x \in S \wedge x \notin T$ \\
\hline Powerset & $x \in \mathbb{P}(S)$ & $x \subseteq S$ \\
\hline Empty set & $x \in \varnothing$ & false \\
\hline
\end{tabular}

Note that $x \notin T$ is a shorthand for $\neg(x \in T)$. Similarly we can use the shorthand $S \neq T$ for $\neg(S=T)$.

\section{Structuring models with machines}

We have already introduced several Event-B constructs such as carrier sets, variables, invariants and events. So is there a way of packaging these into components? A machine is an Event-B component in which the variables, invariants, and events are placed. Carrier sets that are required by a machine are placed 
in a separate component called a context. An Event-B context can also contain constants and axioms. The axioms are predicates that define properties of the carrier sets and the constants. For example, for our building access control example, we might want to model a capacity constraint on the building. We could do this by introducing a constant max_capacity and an axiom stating that max_capacity is greater than zero.

\subsection{Context}

A context with name $C 1$ has the following form:

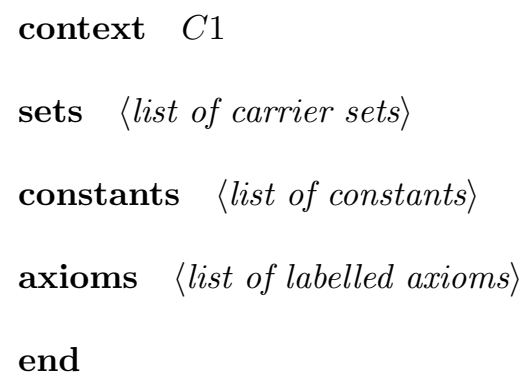

The following example is a context for the building access model which introduces a carrier set and a constant:

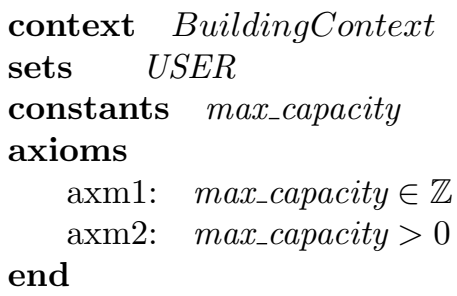

Each axiom in the context is a predicate. For traceability purposes, each axiom in a context is given a unique label (e.g., axm1). The axioms in this context specify that max_capacity is an integer (axm1) whose value is assumed to be greater than zero (axm2).

\subsection{Machine}

In Event-B, a machine defines the dynamic behaviour of a model through events that are guarded by and act on the variables. The events are expected to maintain the invariants; we will see later how this is verified. A machine may see one or more contexts which provide the carrier sets, constants and axioms to be used by the machine. A machine with name $M$ has the following form: 


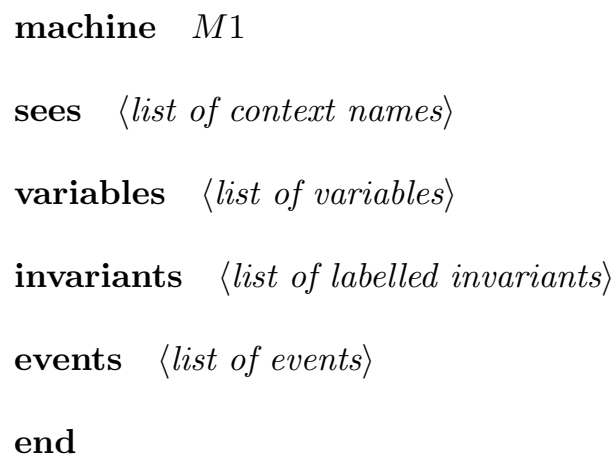

For example, part of the machine for the building access is specified as follows:

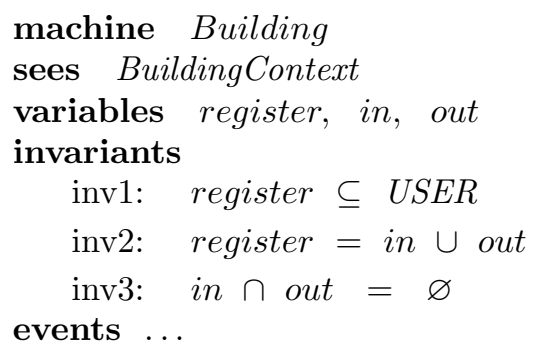

This machine is named Building; it sees the previously defined BuildingContext and it contains three variables. register, in and out. As discussed previously, the invariants specify that registered users are of type USER (inv1), registered users are either inside or outside (inv2) and no user is both inside and outside (inv3).

We postpone treatment of any building capacity constraint to later.

In Section 2 we showed the Enter event which models a user entering the building. Here we present the general syntax of event definitions. Each event of a machine has a name, a list of parameters, a list of guards and a list of actions structured as follows:

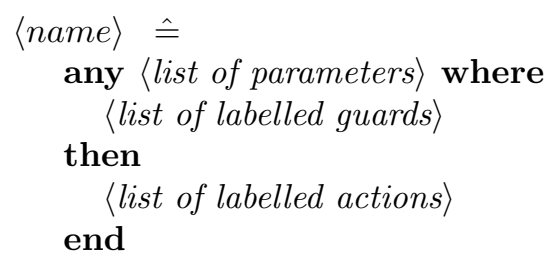

Guards are predicates that specify conditions on the machine variables and the event parameters. Each action assigns a value to a machine variable and has the form:

$$
\langle\text { variable }\rangle:=\langle\text { expression }\rangle
$$

For example, here is the Enter event again: 


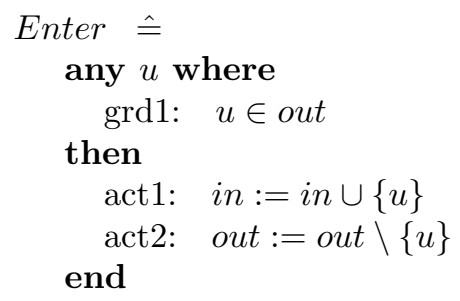

An event may be executed for particular values of the parameters when all its guards are satisfied. When an event is executed, all of the actions of that event are performed simultaneously. Because of the simultaneity, it is not allowed for two different actions in an event to assign to the same variable as this would lead to conflicting updates. As with invariants, the guards and actions are labelled.

\subsection{Preserving Invariants}

When specifying an event, it is important to ensure that the invariants are preserved by its actions. We can assume that the invariants are satisfied prior to execution of the event and we need to demonstrate that the actions do not result in any invariant being violated.

Let us consider whether the Enter event preserves the invariants of the access control model. Invariant inv1 refers to the register variable only and, since none of the actions modify register, this invariant is trivially preserved. Invariant inv 2 is an equation specifying that register is the union of in and out:

$$
\text { register }=i n \cup o u t
$$

The actions of the Enter event modify the variables in right-hand side of the equation but not the left-hand side. However since $u$ is moved from out to $\mathrm{in}$, the overall value on the right-hand side remains unchanged and the equation remains valid. More precisely, the effect of the actions of the Enter event on the invariant can be represented by replacing each variable in the invariant by the expression on the right-hand side of the assignment to that variable, i.e., replace in by in $\cup\{u\}$ and out by out $\backslash\{u\}$, giving:

$$
\text { register }=\underline{(i n \cup\{u\})} \cup \underline{(\text { out } \backslash\{u\})}
$$

The result of replacing in and out are underlined in equation (2). We say that invariant inv2 is preserved when equation (2) follows from equation (1) and the guard of Enter, that is, when proving tequation (2), we can assume that equation (1) holds and that the guards of the Enter event hold. The proof is as follows:

$$
\begin{aligned}
& (\text { in } \cup\{u\}) \cup(\text { out } \backslash\{u\}) \\
= & \text { " } \cup \text { is associative and commutative" } \\
& \text { in } \cup(\text { out } \backslash\{u\}) \cup\{u\}
\end{aligned}
$$




$$
\begin{aligned}
& =\underset{\text { in } \cup \text { out }}{ } \quad \text { grd } 1: u \in \text { out" } \\
& =\underset{\text { register }}{ } \\
& \text { "inv" }
\end{aligned}
$$

Each step in the simple proof is justified either by appealing to a rule of set theory (the first step), by appealing to an event guard (the second step) or to the invariant inv2 (the third step) with the justification indicated by "inverted commas". Both union and intersection are associative and commutative as captured in this table:

\begin{tabular}{|c|c|}
\hline Description & Rule \\
\hline Union associative & $(s \cup t) \cup u=s \cup(t \cup u)$ \\
\hline Union commutative & $s \cup t=t \cup s$ \\
\hline Intersection associative & $(s \cap t) \cap u=s \cap(t \cap u)$ \\
\hline Intersection commutative & $s \cap t=t \cap s$ \\
\hline
\end{tabular}

The second step in the above proof of equation (2) relies on the following simplification rule for sets which states that if $x$ is in set $s$, then subtracting $x$ from $s$ and adding $x$ to the result yields $s$ :

\begin{tabular}{|c|c|}
\hline Description & Rule \\
\hline Simplify & $x \in s \Rightarrow(s \backslash\{x\}) \cup\{x\}=s$ \\
\hline
\end{tabular}

An advantage of the actions of an event being executed simultaneously is that we do not need to consider intermediate states in which invariants might be violated. For example, if act 1 and act 2 were executed sequentially, inv 2 would be violated in between act 1 and act 2 before being re-established by act 2 . To re-iterate: the actions within an event are always executed simultaneously and not sequentially.

We we have shown that the Enter event maintains $i n v 1$ and $i n v 2$. We now consider the remaining invariant, inv3. Invariant inv3 specifies that in and out are disjoint. If we removed action act2 from the Enter event, this would lead to a violation of inv2 as $u$ would end up both in and out. However, since both actions together move $u$ from out to in, their disjointness is preserved. Let us prove this mathematically. Invariant inv2 is:

$$
\text { in } \cap \text { out }=\varnothing
$$


As we have seen, the effect of the actions of the Enter event on the invariant can be represented by replacing each variable in the invariant by the expression on the right-hand side of the assignment to that variable, i.e., replace in by $i n \cup\{u\}$ and out by out $\backslash\{u\}$, giving:

$$
\underline{(i n \cup\{u\})} \cap \underline{(o u t \backslash\{u\})}=\varnothing
$$

The proof of this is captured by the following general rule about sets which states that if two sets are disjoint then removing elements from one and adding them to the other maintains the disjointness:

\begin{tabular}{|c|cc|}
\hline Description & \multicolumn{2}{|c|}{ Rule } \\
\hline Keep disjoint & $s \cap t=\varnothing \quad \Rightarrow \quad(s \backslash r) \cap(t \cup r)=\varnothing$ \\
\hline
\end{tabular}

\subsection{Machine Initialisation}

Every machine has a special event (named initialisation) that initialises the machine variables. The acccess control machine is initialised by setting all three variables to be empty:

$$
\begin{aligned}
& \text { initialisation } \hat{=} \\
& \text { act1: in }:=\varnothing \\
& \text { act2: } \text { out }:=\varnothing \\
& \text { act3: register }:=\varnothing
\end{aligned}
$$

An initialisation event has no guards nor parameters and the assignment expressions (right-hand side) cannot refer to the machine variables. This is because no assumptions can be made about the values of the variables prior to initialisation. The initialisation should establish the invariant, i.e., the values assigned to the variables together should satisfy the invariants. In this case, all three invariants are trivially established, i.e.,

$$
\begin{aligned}
& \varnothing \subseteq U S E R \\
& \varnothing \cap \varnothing=\varnothing \\
& \varnothing=\varnothing \cup \varnothing
\end{aligned}
$$

\subsection{Other Access Control Events}

We now look at some of the other events for access control: exiting the building, registering a new user and de-registering a user. The Exit event is the opposite of the Enter event: the user should be in the building and is moved from in to out: 


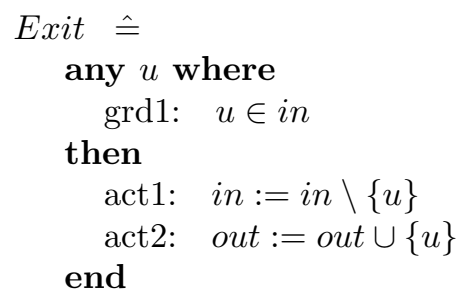

This event maintains the invariants based on similar arguments that we used previously for the Enter event.

When registering a user, we need a 'fresh' value to represent the new user, i.e, a value that is not already in the set register. This fresh value is then added to the set of registered users. The event could be specified as follows:

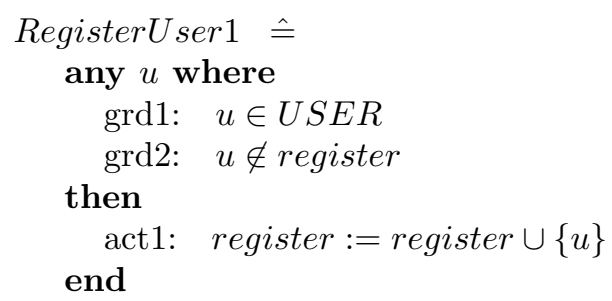

The first guard gives a type to $u$ while the second guard ensures that $u$ is fresh. Let us consider whether the action violates any invariants. It turns out that the action violates the equation of inv2 (register $=i n \cup$ out): it expands the lefthand side without expanding the right-hand side. We can resolve this by adding an action that also expands the right-hand side of the equation. We can do this by adding $u$ to in or to out. In this case, it makes more sense to add $u$ to out rather than in, as we would not expect that the new user would end up inside the building immediately at the point at which they are registered. Thus, an improved version of the event is specified as follows:

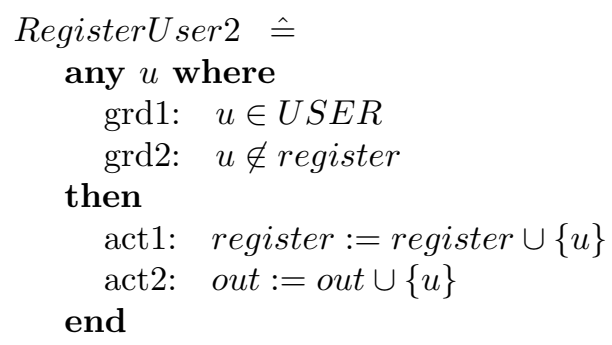

This specification of the user registration does maintain inv3. Let us prove this mathematically. Invariant $i n v 3$ is:

$$
\text { register }=\text { in } \cup \text { out }
$$

The actions of the event modify this to the following equation:

$$
\underline{\text { register } \cup\{u\}}=\text { in } \cup \underline{(\text { out } \cup\{u\})}
$$


We prove that this equation follows from the invariant:

$$
\begin{aligned}
& \text { register } \cup\{u\} \\
= & \text { "inv3" } \\
& (\text { in } \cup \text { out }) \cup\{u\} \\
= & \text { " } \cup \text { is associative" } \\
& \text { in } \cup(\text { out } \cup\{u\})
\end{aligned}
$$

A user who is already registered may be de-registered by removing them from register. Removing $u$ from register without removing $u$ from in or out will lead to a violation of $i n v 2$. One solution is to remove $u$ from both in and out leading to the following specification of the event for de-registering:

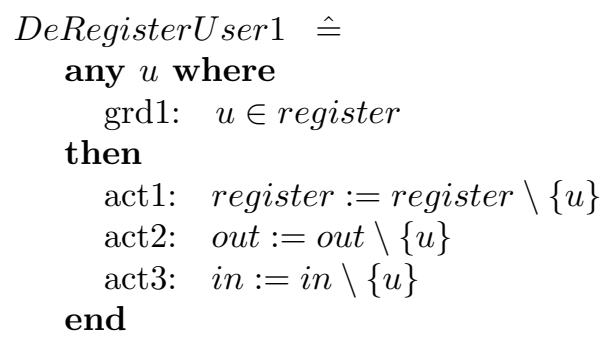

This specification will preserve $i n v 2$ since $u$ is removed from both sides of the equation. This event is applicable whether registered user $u$ is inside or outside the building. However, if we consider a building access control system, it probably does not make sense to de-register a user while they are inside the building so we strengthen the guard to specify that $u$ is outside (and registered):

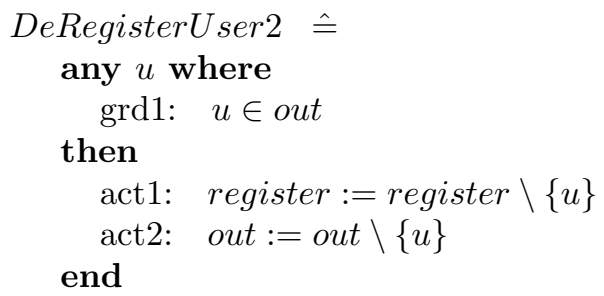

Note that this version does not modify $i n$. If $u$ was a member of $i n$, this would result in a violation of $i n v 2$. However, from the guard of the event we know that $u$ is an element of out and, since in and out are disjoint (inv3), we know that $u$ cannot be an element of $i n$. Thus it is sufficient to remove $u$ from out in order to maintain inv2.

We leave it as an exercise for the reader to prove that DeRegisterUser2 preserves the invariants. The following rules are used in the proofs: 


\begin{tabular}{|c|c|}
\hline Description & Rule \\
\hline Distribute difference & $(s \cup t) \backslash r=(s \backslash r) \cup(t \backslash r)$ \\
\hline Simplify & $x \notin s \Rightarrow s \backslash\{x\}=s$ \\
\hline Keep disjoint & $s \cap t=\varnothing \Rightarrow \quad(s \backslash r) \cap t=\varnothing$ \\
\hline
\end{tabular}

\subsection{Machine behaviour and nondeterminism}

A simple way of thinking about the behaviour of an Event-B machine is as a transition system that moves from one state to another through execution of events. The states of a machine are represented by the different configurations of values for the variables. The variables of a machine are initialised by execution of the special initialisation event. An event is enabled in some state for some parameter values if all of the guards of the event are satisfied. For example, the Enter event in the access control model is enabled for parameter value $u 1$ in any state in which $u 1$ is an element of the variable out.

In any state that a machine can reach, an enabled event is chosen to be executed to define the next transition. If several events are enabled in a state, then the choice of which event occurs is nondeterministic. Also, if an event is enabled for several different parameter values, the choice of value for the parameters is nondeterministic - the choice just needs to satisfy the event guards. For example, in the RegisterUser2 event, the choice of value for parameter $u$ is nondeterministic, with the choice of value being constrained by the guards of the event to ensure that it is a fresh value.

Treating the choice of event and parameter values as nondeterministic is an abstraction of different ways in which the choice might be made in an implementation of the model. For example, if it is an interactive system, the choice might be offered to a user via a graphical interface. If it is an information processing system, the choice might be made by some scheduler. If the machine reaches a state in which no event is enabled, then it is said to be deadlocked.

\section{Finiteness, cardinality and well-definedness}

Previously we considered the possibility of placing a constraint on the the number of users allowed inside the building at any one time. We could represent this as an invariant specifying that the number of elements in the set in is bounded by the constant max_capacity. In set theory, the number of elements in a set is called its cardinality and in Event-B this is written as $\operatorname{card}(S)$. For example,

$$
\operatorname{card}(\{a, b, c\})=3 .
$$


However a word of caution: cardinality is only defined for finite sets. If $S$ is an infinite set, then $\operatorname{card}(S)$ is undefined. Whenever we use the $\operatorname{card}$ operator, we must ensure that it is only applied to a finite set. This issue of well-definedness applies to some other operators as well. For example, division by zero is not well-defined and when using division we must ensure that the divisor is not zero.

As is standard in set theory, sets in Event-B may be finite or infinite. For example, the set of integers is infinite. A carrier set defined in a context is infinite unless we explicitly specify that it is finite. Naturally, an enumerated set, e.g., $S=\{a, b, c\}$, is finite. We can specify that a set $S$ is finite using the predicate finite $(S)$. In the building access system, we would expect the set of people who are inside the building to be finite which we write as finite(in). Initially in is empty and thus finite. The only way of expanding the set $i n$ is through the Enter event which adds one user at a time. Thus the set in can never become infinite.

To model the finiteness and capacity constraints on the access control, we extend the set of invariants of the machine as follows:

\section{invariants}

$$
\begin{array}{ll}
\text { inv4: } & \text { finite }(\text { in }) \\
\text { inv5: } & \text { card }(\text { in }) \leq \text { max_capacity }
\end{array}
$$

In $i n v 5, \operatorname{card}(i n)$ is well-defined since we know that in is finite from inv4. Considering preservation of inv4, the only event that expands in is the Enter event and it maintains the finiteness of in (inv4) by the argument outlined above. For inv5, max_capacity is a constant so cannot decrease during execution of the machine so we only need to consider events that might cause card(in) to increase. As we have already said, Enter is the only event that expands in and thus increases $\operatorname{card}($ in $)$. The Enter event as previoulsy specified places no constraint on the number of people already in the building so we need to strengthen it by adding guard grd2 as follows:

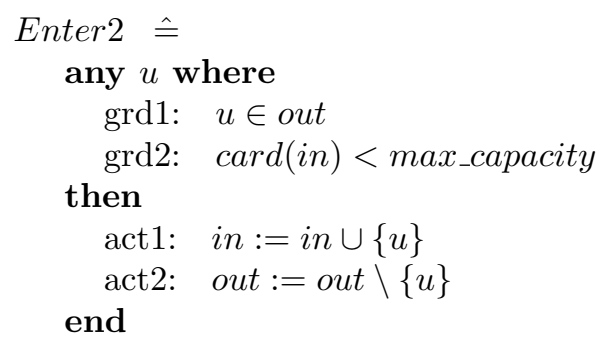

Note that $\operatorname{grd} 2$ requires $\operatorname{card}(\mathrm{in})$ to be strictly less that max_capacity in order to ensure that the size of the resulting value for in is less than or equal to max_capacity.

The following table summaries the finiteness and cardinality operators we have just introduced. The table also includes a column to indicate when a predicate or expression is well-defined: 


\begin{tabular}{|l|l|l|l|}
\hline Name & Operator & Meaning & Well-definedness \\
\hline Finite & finite $(S)$ & set $S$ is finite & true \\
\hline Cardinality & $\operatorname{card}(S)$ & number of elements in set $S$ & finite $(S)$ \\
\hline
\end{tabular}

Note that some of our definition tables do not have a well-definedness column. In these cases the predicate or expression is always well-defined.

The following rules about finiteness and cardinality are used to prove that inv 4 and inv5 are preserved by the events:

\begin{tabular}{|c|c|}
\hline Description & Rule \\
\hline Finite union & finite $(s) \wedge$ finite $(t) \Rightarrow$ finite $(s \cup t)$ \\
\hline Finite difference & finite $(s) \Rightarrow$ finite $(s \backslash t)$ \\
\hline Increase card & $x \notin s \Rightarrow \operatorname{card}(s \cup\{x\})=\operatorname{card}(s)+1$ \\
\hline Decrease card & $x \in s \Rightarrow \operatorname{card}(s \backslash\{x\})=\operatorname{card}(s)-1$ \\
\hline
\end{tabular}

\section{Introducing relations}

We have seen how sets can be used to model access control for a building. We introduced a carrier set to represent users but we did not introduce a carrier set to represent buildings. The reason for not introducing buildings is that our model was intended for a single building and the identity of that building was implicit. Let us consider generalising our modelling of access control to a system with multiple buildings. For this we introduce a carrier set representing buildings so that we can distinguish different buildings. Figure 8 illustrates the two distinct carrier sets, one for users and the other for buildings.

Rather than allowing registered users to enter any building, we would like to model a more fine-grained control over which buildings a user is allowed to enter. This is illustrated in Figure 9 which represents a permission relation between users and buildings. An arrow from a user to a building indicates that that particular user has permission to enter that building. For example, in Figure 9, user $u 1$ has permission to enter two of the buildings, $b 1$ and $b 2$. Figure 9 represents three different sets: a set of users, a set of buildings and a set of arrows between users and buildings. Mathematically an arrow from user $u$ to building $b$ is represented by a pair of elements, written $u \mapsto b$. A relation is represented 

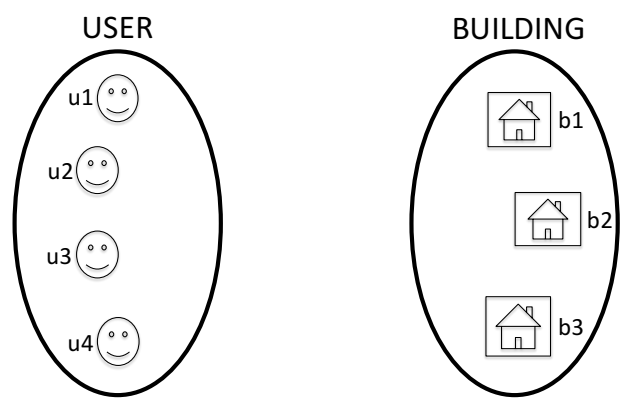

Fig. 8. Distinct carrier sets

by a set of pairs, for example, the permission relation of Figure 9 is represented by the following set $P$ of pairs:

$$
P=\{u 1 \mapsto b 1, \quad u 1 \mapsto b 2, \quad u 2 \mapsto b 1, \quad u 2 \mapsto b 3, \quad u 4 \mapsto b 2, \quad u 4 \mapsto b 3\}
$$

The permission model demonstrates that a relation allows us to connect distinct carrier sets. Management of relationships between different kinds of entities is a key role of many computerised systems, including access control, business systems, information systems and communications systems. Thus relations are a useful mathematical structure for modelling such systems.

A pair $u \mapsto b$ has a first element $u$ and a second element $b$. Given a set of pairs, it is useful to refer to the set of the first elements of all pairs, called the domain, and the set of second elements, called the range. For the example relation $P$ above, we have

$$
\begin{aligned}
\operatorname{dom}(P) & =\{u 1, u 2, u 4\} \\
\operatorname{ran}(P) & =\{b 1, b 2, b 3\}
\end{aligned}
$$

Here, $\operatorname{dom}(P)$ represents the set of users who have permission to enter some building while $\operatorname{ran}(P)$ represents the set of buildings for which some user has permission to enter.

Figure 9 labels the permission relation as many-to-many. This means that many different domain elements can be mapped to the same range element, e.g., $u 1$ and $u 2$ are both mapped to $b 1$, and also that the same domain element can be mapped to many different range elements, e.g., $u 1$ is mapped to both $b 1$ and $b 2$.

As well as modelling the permission relation, we can also model the current location of a user using a relation as illustrated in Figure 10. We would not expect a user to be located in more than one building at a time and thus the location relation is required to be a many-to-one relation, meaning that a domain element can be mapped to exactly one range element rather than many. A manyto-one relation still allows many different domain elements to be mapped to the same range element, e.g., $u 2$ and $u 4$ are both located in the same building in 


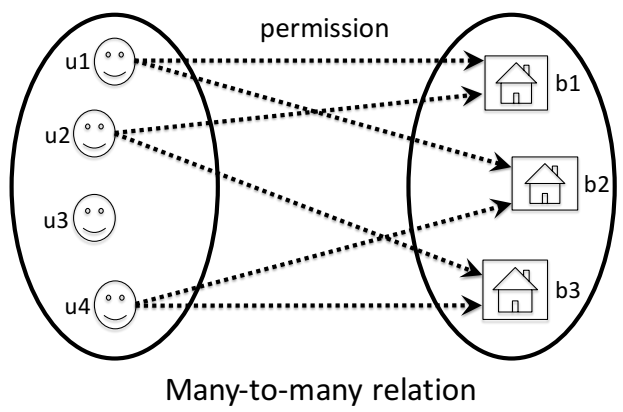

Fig. 9. Permission relation

Figure 10. Many-to-one relations are also called functions and are covered in more detail in Section 7.3.

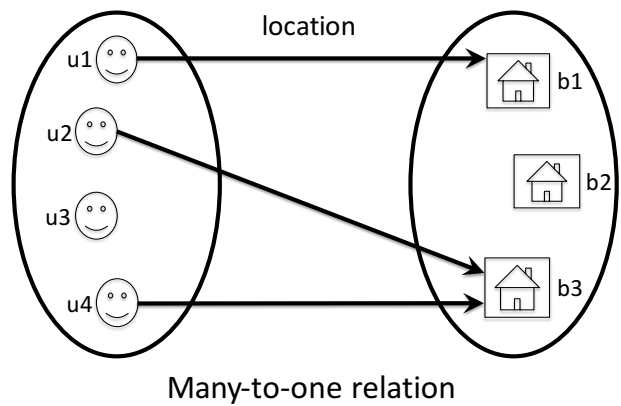

Fig. 10. Location relation

Since the permission and location relations are themselves sets, we can formulate a connection between them. For an access control system we require that if a user is located in a building, then they have permission to be in that building. This requirement is represented by specifying that location is a subset of permission, i.e., any pair in the location relation is also a pair of the permission relation. The connection between the two relations is illustrated in Figure 11 where location is clearly a subset of permission.

A many-to-one relation is a special case of a many-to-many relation. A further special case is a one-to-one relation in which each domain element is related to exactly one range element and each range element is related to exactly one domain element. This is illustrated in Figure 12 where the location relation is such that users are mapped one-to-one with buildings, i.e., no two users are located in the same building. If we required single occupancy for the buildings, 


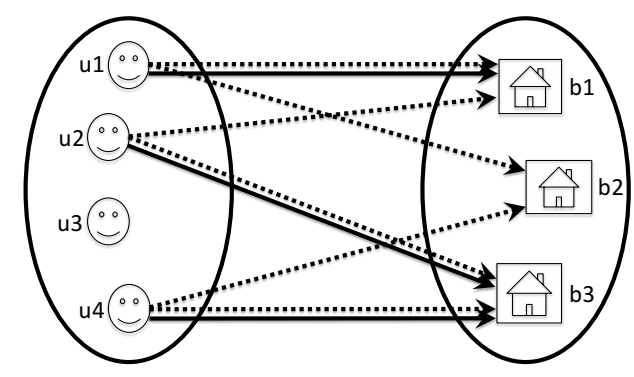

invariant: location $\subseteq$ permission

Fig. 11. Location satisfies permission

then we could represent this with an invariant specifying that location is one-toone.

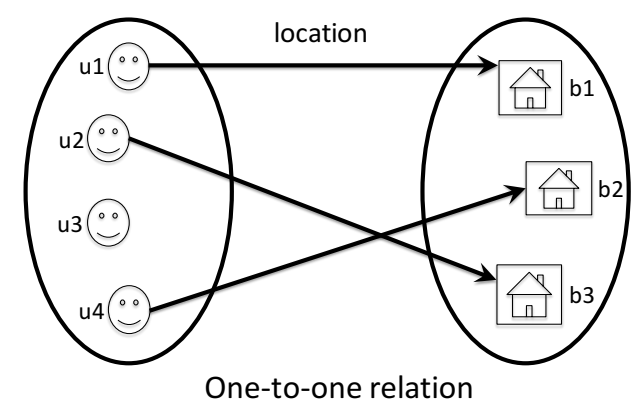

Fig. 12. Location with single occupancy

\section{Cartesian products and relations}

We have seen that an ordered pair is an element consisting of two parts, a first part and a second part, and is written as $x \mapsto y$. Given two sets $S$ and $T$, we can form what is called their Cartesian product. This is the set of all those pairs whose first component is in $S$ and second component is in $T$. The Cartesian product of $S$ with $T$ is written $S \times T$. For example, the Cartesian product of $\{a, b, c\}$ with $\{1,2\}$ is expanded to a set of pairs as follows:

$$
\begin{aligned}
\{a, b, c\} \times\{1,2\}=\{a & \mapsto 1, a \mapsto 2, \\
b & \mapsto 1, b \mapsto 2, \\
c & \mapsto 1, c \mapsto 2\}
\end{aligned}
$$


Here we see, for example, that $a \mapsto 1$ is an element of the Cartesian product since $a$ is in $\{a, b, c\}$ and 1 is in $\{1,2\}$. More generally $x \mapsto y$ is an element of $S \times T$ when $x$ is in $S$ and $y$ is in $T$ as shown in the following table:

\begin{tabular}{|c|c|c|}
\hline Name & Predicate & Definition \\
\hline Cartesian product & $x \mapsto y \in S \times T$ & $x \in S \wedge y \in T$ \\
\hline
\end{tabular}

The following derivation shows that the product of any set with the empty set is itself empty $(S \times \varnothing=\varnothing)$ :

$$
\begin{aligned}
& x \mapsto y \in S \times \varnothing \\
= & \text { "Definition of } \times " \\
& x \in S \wedge y \in \varnothing \\
= & \text { "Definition of } \varnothing " \\
& x \in S \wedge \text { false } \\
= & \text { "Logic" } \\
& \text { false } \\
= & \text { "Definition of } \varnothing " \\
& x \mapsto y \in \varnothing
\end{aligned}
$$

\subsection{Type constructors and structured types}

In Section 3.1, we saw that the powerset operator is used to define the type of a set. The powerset operator can be used to construct a type $\mathbb{P}(T)$ from a type $T$ so we refer to it as a type constructor. Similarly, Cartesian product is a type constructor: the type $S \times T$ is constructed from the types $S$ and $T$. A structured type is a type formed using a type constructor such as $\mathbb{P}$ or $\times$.

- Powerset $(\mathbb{P})$ is the type constructor for sets.

- Cartesian product $(\times)$ is the type constructor for ordered pairs.

In Event-B, constants, variables, parameter and expressions have a type and these types come in three forms

- Basic type: integer $(\mathbb{Z})$, Boolean.

- Carrier set, e.g., USER, BUILDING.

- Structured type: $\mathbb{P}(S), \quad S \times T$.

The type constructors can be nested and combined to form more complex structured types such as:

- Set of sets: $\mathbb{P}(\mathbb{P}(T))$

- Set of pairs: $\mathbb{P}(S \times T)$

- Pair of sets: $\mathbb{P}(S) \times \mathbb{P}(T), \quad S \times \mathbb{P}(T), \quad \mathbb{P}(S) \times T$ 
The following table presents some example expressions and their corresponding structured type:

\begin{tabular}{|c|c|}
\hline Expression & Type \\
\hline$\{5,6,3\}$ & $\mathbb{P}(\mathbb{Z})$ \\
\hline $4 \mapsto 7$ & $\mathbb{Z} \times \mathbb{Z}$ \\
\hline$\{5,6,3\} \mapsto 7$ & $\mathbb{P}(\mathbb{Z}) \times \mathbb{Z}$ \\
\hline$\{4 \mapsto 8,3 \mapsto 0,2 \mapsto 9\}$ & $\mathbb{P}(\mathbb{Z} \times \mathbb{Z})$ \\
\hline
\end{tabular}

\subsection{Relations}

Through the permission example (Figure 9) we have seen that a relation is modelled as a set of pairs, i.e., a structured type formed using both the $\times$ and $\mathbb{P}$ constructors. Because this structured type is a useful modelling construct, it is given its own symbol in Event-B: we write $S \leftrightarrow T$ as a shorthand for $\mathbb{P}(S \times T)$. The following table provides the definition of the relation arrow:

\begin{tabular}{|c|c|c|}
\hline Name & Predicate & Definition \\
\hline Relation & $r \in S \leftrightarrow T$ & $r \in \mathbb{P}(S \times T)$ \\
\hline
\end{tabular}

For the access control example, we may specify that the permission variable is a (many-to-many) relation with the following invariant:

$$
\text { invariant permission } \in U S E R \leftrightarrow B U I L D I N G
$$

Here is another example of a relation, named directory, that relates people to phone numbers:

invariant directory $\in$ PERSON $\leftrightarrow N U M B E R$

A possible value for the directory is as follows:

$$
\begin{aligned}
& \text { directory }=\{\text { mary } \mapsto 287573 \text {, } \\
& \text { mary } \mapsto 398620 \text {, } \\
& \text { john } \mapsto 829483 \text {, } \\
& \text { jim } \mapsto 398620\}
\end{aligned}
$$


It is worth pointing out the difference between the two arrow symbols used in representing relations:

$\leftrightarrow$ combines two sets to form a set.

$\mapsto$ combines two elements to form an ordered pair.

We already introduced the domain and range of a relation. These are defined by the following table:

\begin{tabular}{|c|c|c|}
\hline Name & Predicate & Definition \\
\hline Domain & $x \in \operatorname{dom}(R)$ & $\exists y \cdot x \mapsto y \in R$ \\
\hline Range & $y \in \operatorname{ran}(R)$ & $\exists x \cdot x \mapsto y \in R$ \\
\hline
\end{tabular}

For the example directory shown above, we have:

$$
\begin{aligned}
\operatorname{dom}(\text { directory }) & =\{\text { mary }, j o h n, j i m\} \\
\operatorname{ran}(\text { directory }) & =\{287573,398620,829483\}
\end{aligned}
$$

Note that when we declare a constant or variable to be a relation between two sets, as well as defining its type, we are implicitly constraining the domain and range of the relation: Suppose we have $s \subseteq S$ and $t \subseteq T$ and we declare $r \in s \leftrightarrow t$, then it follows that

$$
\begin{gathered}
r \in \mathbb{P}(S \times T) \\
\operatorname{dom}(r) \subseteq s \\
\operatorname{ran}(r) \subseteq t
\end{gathered}
$$

\subsection{Functions}

From Figure 10 we saw that the location relation should be a many-to-one relation, i.e., each user is located in at most one building at any moment. The many-to-one property means that if a user $u$ is in the domain of location, then that user is mapped to a single building by the location relation. In that case, we can write location $(u)$ to refer to the building that $u$ is located in. For example, from Figure 10, we have:

$$
\begin{aligned}
& \operatorname{location}(u 1)=b 1 \\
& \operatorname{location}(u 2)=b 3 \\
& \operatorname{location}(u 4)=b 3
\end{aligned}
$$

If a user $u$ is not in the domain of location, then location $(u)$ is not well-defined. For example, from Figure 10, u3 is not in the domain of location therefore location (u3) is not well-defined. 
In general, a many-to-one relation $f$ is said to be functional. This is written as $f \in S \rightarrow T$ and means that every element in the domain of $f$ is mapped to exactly one element in the range. The functionality property is specified mathematically by stating that if a domain value $x$ is mapped to range value $y$, then $x$ cannot be mapped to any other range value $y^{\prime}$. This is shown in the following table:

\begin{tabular}{|c|c|c|}
\hline Name & Predicate & Definition \\
\hline Partial Function & $f \in S \rightarrow T$ & $\begin{array}{l}f \in S \leftrightarrow T \wedge \\
\forall x, y, y^{\prime} \cdot x \mapsto y \in f \wedge y^{\prime} \neq y \\
\quad \Rightarrow \quad x \mapsto y^{\prime} \notin f\end{array}$ \\
\hline
\end{tabular}

Note that when we declare $f \in S \rightarrow T$ we say that $f$ is a partial function. It is said to be partial because there may be values in the set $S$ that are not in the domain of $f$. For example, from Figure 10, $u 3$ is in USER but is not in the domain of location. A relation is said to be a total function from $S$ to $T$ when it is a partial function and its domain is exactly $S$ :

\begin{tabular}{|c|c|c|}
\hline Name & Predicate & Definition \\
\hline Total Function & $f \in S \rightarrow T$ & $f \in S \rightarrow T \wedge \operatorname{dom}(f)=S$ \\
\hline
\end{tabular}

We have seen that we can write location $(u 1)$ since location is functional. In general, when a relation $f$ is functional, we can treat it as a mathematical function and write $f(x)$ for the value that $x$ is mapped to. For $f(x)$ to be welldefined, two conditions must hold: $f$ must be functional and $x$ must be in the domain of $f$. This is shown in the following definition:

\begin{tabular}{|c|c|c|c|}
\hline Name & Expression & Meaning & Well-definedness \\
\hline Function Application & $f(x)$ & $\begin{array}{l}f(x)=y \\
\Leftrightarrow x \mapsto y \in f\end{array}$ & $\begin{array}{l}f \in S \rightarrow T \quad \\
x \in \operatorname{dom}(f)\end{array}$ \\
& & & \\
\hline
\end{tabular}

This definition uses the if and only if $(\Leftrightarrow)$ logical operator: $P \Leftrightarrow Q$ is short for $P \Rightarrow Q \wedge Q \Rightarrow P$.

\section{Access control specification}

Now that we have explained relations and functions, we will make use of them to construct an Event-B specification of access control for multiple buildings. We start by presenting the high-level requirements in an informal way. As already stated, computer-based system is designed to satisfy some requirements in the real world and it is usual to express system requirements in natural language. 
Documentation of the requirements in natural language will guide the construction of the Event-B specification and will also provide a form of "sanity check" against which to validate the Event-B specification. It helps understanding if we try to describe the intended purpose of the system being designed in a concise way. For the access control system this is as follows:

Purpose: The purpose of the access control system is to ensure that users may be in a building only if they have permission to be in that building.

We provide a more detailed list of requirements, giving each one a label so that we can refer to it later. In each of the following requirements "the system" refers to the access control system:

- FUN1: The system shall maintain a register of recognised users and shall provide operations for managing the user register.

- FUN2: The system shall maintain a register of protected buildings and shall provide operations for managing the building register.

- FUN3: The system shall maintain the permissions for each user, determining the building they are allowed to enter, and shall provide operations for managing the permissions.

- FUN4: The system shall allow a user to enter a building provided they have permission.

- FUN5: The system shall allow a user to exit a building without constraint.

- ASM1: A user will be in at most one building at any time.

- ASM2: A user cannot move directly from one building to another building.

Most of these requirements are functional requirements ${ }^{1}$, that is, requirements defining the intended function of the system. The last requirements in the list are assumptions about the environment in which the system is operating, e.g., we assume that there is a physical constraint on users which means they cannot be in more than one building at any time.

We referrred to the requirements as high-level. By this we mean there is not necessarily enough detail in the requirements to build the system. For example, FUN4 does not provide detail on how a user would enter a building or how they might be prevented from entering. Nonetheless, we will see that it is still feasible and useful to make a formal analysis of the high-level requirements in Event-B.

\subsection{Set and relations for access control}

From the requirements FUN1 and FUN2 we identify two kinds of entity in the system, users and buildings. These give rise to two carrier sets for our specification of the system as defined in the following context:

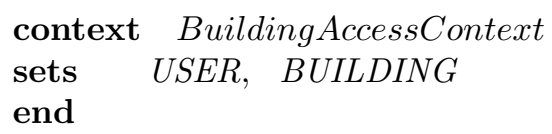

${ }^{1}$ Not to be confused with a functional (many-to-one) relation! 
Having identified the carrier sets, we consider what set variables to include in the model, i.e., variables that are subsets of a carrier set. Looking at FUN1, we see that a variable set of registered users is required. We will call this variable user, where user $\subseteq$ USER. Similarly, FUN2 suggests a variable set of buildings so we introduce a variable building $\subseteq$ BUILDING. These two set variables are specified in an Event-B machine as follows:

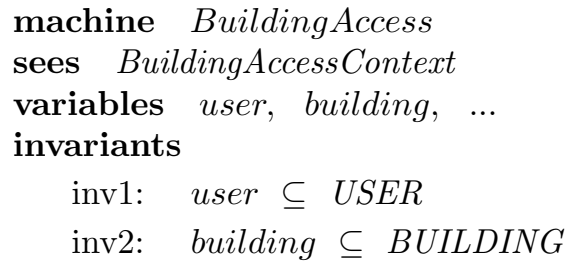

Naming convention: Although it is not required by the Event-B language, we will use all UPPER case letters for names of carrier sets. When a model has multiple carrier sets representing different kinds of entity, we will use a lower case version of a carrier set name for the variable corresponding to the set of instances of that entity. For example, the user entity is represented by the carrier set USER and the set of instances (i.e., the register users) is represented by the variable user. While the carrier set is fixed, the instance set may be expanded or reduced through execution of events.

We also want to identify any required relational variables for our specification. FUN3 suggests a relation to represent user permissions, while FUN4 suggests a relation to represent user location. The diagrams in Figures 9 and 10 illustrate the permission and location relations between users and buildings. These diagrams are useful for illustrating specific instances of relations but they do not provide a general representation. To illustrate relations between sets more generally we use the class diagram shown in Figure 13. A class diagram is a construct from object oriented design that is used to represent classes and associations between classes. In Figure 13, the sets are represented as classes (the

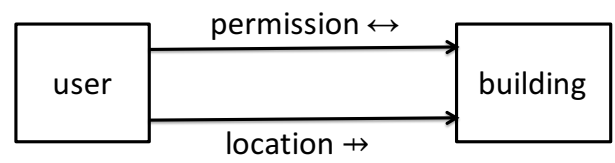

Fig. 13. Relations for access control

boxes) while the relations are presented as associations (the arrows). An association represents a relation between the indicated sets. We place the relevant mathematical symbol next to the name of the relation to indicate its nature 
(many-to-many, many-to-one, etc). Thus Figure 13 indicates that permission is a relation between user and building:

$$
\text { permission } \in \text { user } \leftrightarrow \text { building }
$$

Because of ASM1, Figure 13 indicates that location is a partial function from user to building, giving rise to the following mathematical specification:

$$
\text { location } \in \text { user } \rightarrow \text { building }
$$

From the requirements, and with the aid of a class diagram, we have identified two kinds of variables for our Event-B specification:

- Set variables: user, building

- Relation variables: permission, location

The full list of variables and corresponding invariants is specified as follows:

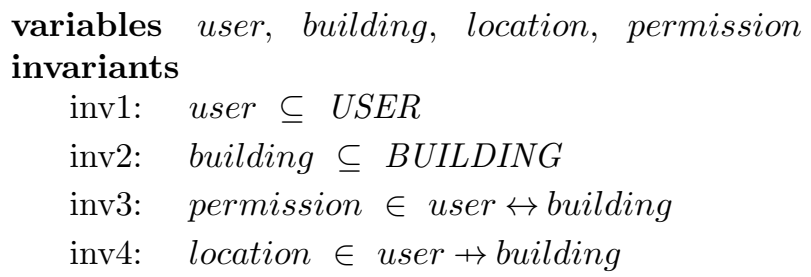

It is important to observe that invariants inv 3 and inv 4 specify constraints between multiple variables (as well as defining the types of the relation variables). For example, included in inv 3 is the constraint that the domain of permission is included in user. This means that the system only maintains permission information for registered users. The range of permission is constrained to be included in building which means that any permissions that a user has can only be for registered buildings. Requirement FUN3 does not precisely state these two constraints though FUN3 could be interpreted as requiring that permission is only between registered users and registered buildings. In the mathematical representation, the constraints are specified precisely. Similarly, inv 4 specifies that only registered users may be located in buildings and those buildings must be registered.

Our access control specification contains two relation variables and we consider whether we can identify an invariant that constrains the connection between these two variables. In fact we have already identified such an invariant in Figure 11 where location is required to be included in permission. Thus our model has one additional invariant:

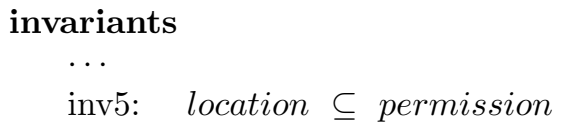


Invariant inv5 addresses FUN4, the main access control requirement.

The list of invariants for the BuildingAccess machine may be classified into three kinds:

1. Constraints between set variables (inv1, inv2).

2. Constraints between a relational variable and set variables (inv3, inv4).

3. Constraints between relational variables (inv5).

The first two kinds of invariant can often be easily identified from a class diagram derived from the requirements. The class diagram is constructed by identifying the main entities suggested by the requirements (e.g., USER and BUILDING) and the relevant relationships between them (e.g. permission and location). The third kind of invariant does not always follow directly from a class diagram and may come directly from the requirements. In Figure 13, because permission and location have the same source and target, the question of whether one is a subset of the other is suggested.

From the requirements, we have identified carrier sets, variables and invariants but the requirements also suggest events to be included in the Event-B specification. Here we identify a list of events by systematically reviewing each requirement:

- FUN1 : Suggests RegisterUser and DeRegisterUser events.

- FUN2 : RegisterBuilding and DeRegisterBuilding.

- FUN3 : AddPermission and RemovePermission.

- FUN4 : EnterBuilding.

- FUN5 : ExitBuilding.

- ASM1 : EnterBuilding.

- ASM2 : EnterBuilding.

As can be seen with the EnterBuilding event, it is sometimes the case that different requirements will give rise to the same event. This is because the requirements may describe different aspects of the behaviour represented by the event. For example, both FUN4 and ASM1 put constraints on when a user may enter a building.

\subsection{Expansion events}

When a specification involves sets, it is common to have events for expanding sets (e.g., RegisterUser) and reducing sets (e.g., DeRegisterUser). We look at the expansion events first. Events to specify registration of users and buildings are similar to the registration event presented in Section 4.5:

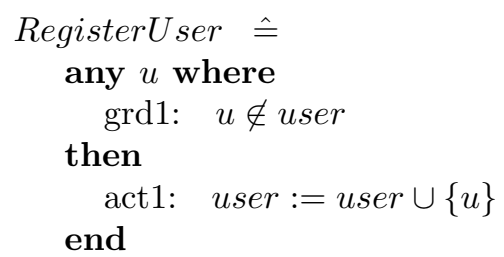




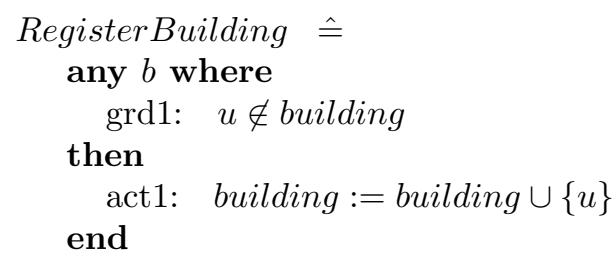

Notice that we omitted a guard specifying that parameter $b$ is an element of $B U I L D I N G$. This is because the type of $b$ can be inferred from grd 1 since the set building has type $\mathbb{P}(B U I L D I N G)$. Similarly for the RegisterUser event.

The AddPermission event gives a registered user $b$ permission to enter a registered building $b$ by adding the ordered pair $u \mapsto b$ to the permission relation:

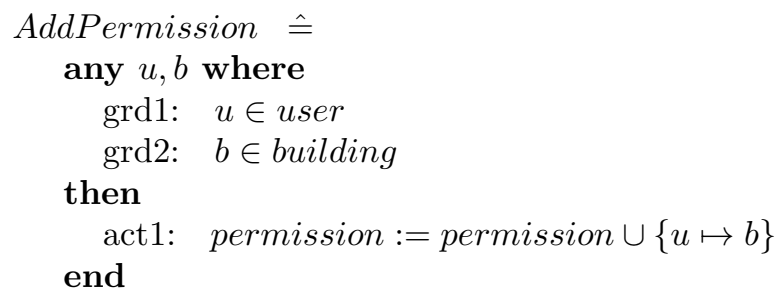

The guards of this event are required in order to preserve invariant inv3 which constrains the domain and range of the permission relation. For example, if $\operatorname{grd} 1$ was $u \in U S E R$ instead then the event might violate the invariant by giving permission to a non-registered user.

Here is an alternative version of the event that adds a set of buildings $b s$ to the users permission rather than a single building:

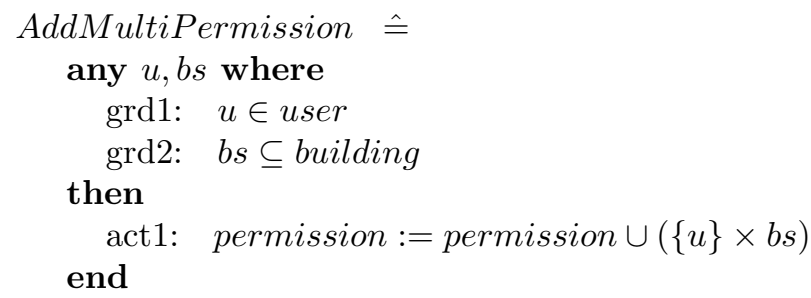

The expression $\{u\} \times b s$ defines a relation that maps $u$ to each element in $b s$. The following rules are used to prove that inv 3 is preserved: 


\begin{tabular}{|c|c|}
\hline Description & Rule \\
\hline $\begin{array}{l}\text { Product } \\
\text { Relation }\end{array}$ & $\begin{array}{l}s \subseteq S \wedge \\
t \subseteq T \\
\quad \Rightarrow \quad s \times t \in S \leftrightarrow T\end{array}$ \\
\hline $\begin{array}{c}\text { Union } \\
\text { Relation }\end{array}$ & $\begin{array}{l}q \in S \leftrightarrow T \wedge \\
r \in S \leftrightarrow T \\
\quad \Rightarrow \quad(q \cup r) \in S \leftrightarrow T\end{array}$ \\
\hline
\end{tabular}

The event modelling a user entering a building is parameterised by the entering user and the building they are entering:

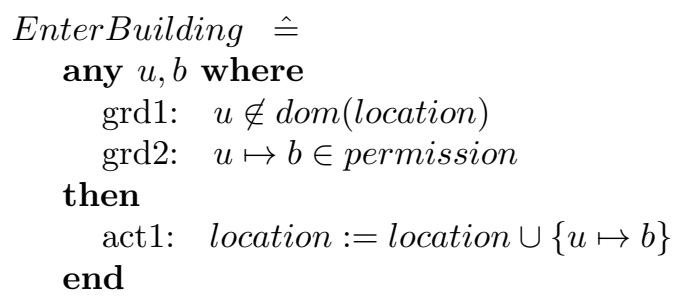

From ASM2 we expect that a user is not located in any building when they try to enter a building hence $\operatorname{grd} 1$ which specifies that $u$ is not in the domain of location. From FUN4, we identify grd2 which specifies that $u$ has permission to enter $b$. The effect of act 1 is to add the ordered pair $u \mapsto b$ to location.

The invariants that the EnterBuilding event affects are inv 4 and inv5. Invariant inv5, which specifies that location is included in permission, is maintained because of grd2. Invariant inv 4 specifies that location is functional. Adding a mapping for $u$ to location maintains the functionality of location because grd 1 specifies that $u$ is not already mapped to any buildings. Without grd1, the event could violate the functionality as $u$ might end up being mapped to more than one building in location. The following rule about expanding a partial function captures this property. It states that if $f$ is functional and $x$ is not in the domain of $f$, then $f \cup\{x \mapsto y\}$ is also functional:

\begin{tabular}{|l|l|}
\hline Description & \multicolumn{1}{|c|}{ Rule } \\
\hline Function & $f \in S \rightarrow T \wedge$ \\
Extension & $\begin{array}{l}x \notin \operatorname{dom}(f) \wedge \\
\\
\end{array}$ \\
& $\Rightarrow \quad(f \cup y \in S \times T$ \\
& \\
\hline
\end{tabular}




\subsection{Reduction events and domain subtraction}

We have already seen how we can reduce sets using the set difference operator. We can use this to model a user $u$ exiting a building $b$ as follows:

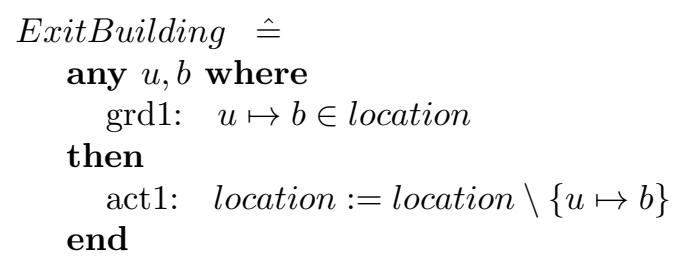

Alternatively, we can use an operator on relations called domain subtraction to model a user exiting a building. Domain subtraction, written $A \triangleleft R$, takes two arguments, a relation a relation $R \in S \leftrightarrow T$ and a set $A \subseteq S$, and removes those pairs from $R$ whose first part is in $A$. This is illustrated by the following equation which shows the result of domain subtracting a set containing a user from an example of the location relation:

$$
\{u 2\} \quad \forall\{u 1 \mapsto b 1, u 2 \mapsto b 3, u 4 \mapsto b 3\}=\{u 1 \mapsto b 1, u 4 \mapsto b 3\}
$$

Here we see that the mapping from $u 2$ to $b 3$ is removed to give the reduced set on the right-hand side. The general definition of the operator is as follows:

\begin{tabular}{|c|c|c|}
\hline Name & Predicate & Definition \\
\hline $\begin{array}{c}\text { Domain } \\
\text { Subtraction }\end{array}$ & $x \mapsto y \in A \triangleleft R$ & $x \mapsto y \in R \wedge x \notin A$ \\
\hline
\end{tabular}

Here is a specification of the ExitBuilding event that has just one parameter, the user $u$. It removes $u$ from the location function using domain subtraction:

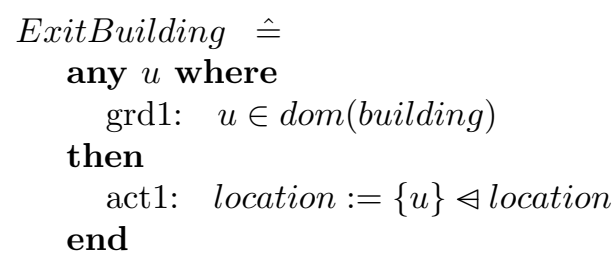

This event preserves the permission invariant (inv5) and the functionality of location (inv3). This follows from the following rules which show that domain subtraction reduces a relation and that inclusion preserves functionality:

\begin{tabular}{|c|c|}
\hline Description & Rule \\
\hline $\begin{array}{c}\text { Domain Subtract } \\
\text { Inclusion }\end{array}$ & $(A \triangleleft R) \subseteq R$ \\
\hline Inclusion & $\begin{array}{c}f \in S \rightarrow T \wedge \\
\text { Functional } \\
\end{array}$ \\
& $\Rightarrow g \in f \in S \rightarrow T$ \\
\hline
\end{tabular}




\subsection{Invariant violation}

While exiting a building causes no problems from the point of view of invariant preservation, removing permissions can be problematic. Consider the following specification of an event that removes all permissions for a user $u$ using the domain subtraction operation:

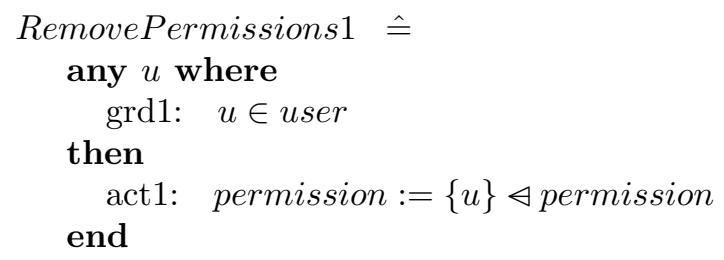

Here is a reminder of the permission inclusion invariant:

$$
\text { inv5: location } \subseteq \text { permission }
$$

Action act 1 of the RemovePermissions 1 event results in the following modified version of $i n v 5$ :

$$
\text { location } \subseteq \underline{\{u\} \varangle \text { permission }}
$$

This does not follow from inv5. The problem is that we are reducing the righthand side of this set inclusion without reducing the left-hand side. If the user $u$ was located in a building and we remove all their permissions, then after executing the RemovePermissions1 event, $u$ would still be in a building but they would no longer have permission to be there, thus violating inv5!

\subsection{Fixing the violation}

One solution to this invariant violation problem would be to add an action to also remove the user from whatever building they are in by removing them from the domain of location as well:

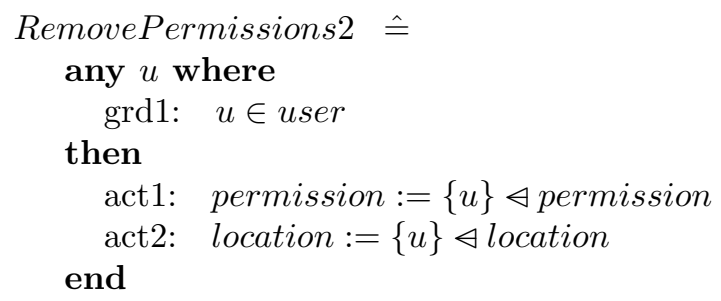

With this version of the event, the modified invariant becomes:

$$
\underline{\{u\} \triangleleft \text { location } \subseteq} \underline{\{u\} \triangleleft \text { permission }}
$$

This inclusion follows from invariant inv5 which means that RemovePermissions2 preserves inv5. Mathematically this is because domain subtraction is monotonic. In general, an operation $o p$ is said to be monotonic when it preserves inclusion between sets: 


\begin{tabular}{|c|c|}
\hline Name & Definition \\
\hline Monotonic & $S \subseteq T \Rightarrow$ op $(S) \subseteq$ op $(T)$ \\
\hline
\end{tabular}

\begin{tabular}{|c|c|}
\hline Description & Rule \\
\hline $\begin{array}{c}\text { Domain Subtract } \\
\text { Monotonic }\end{array}$ & $R \subseteq Q \Rightarrow A \triangleleft R \subseteq A \triangleleft Q$ \\
\hline
\end{tabular}

Another way of ensuring that inv5 is maintained would be to allow permissions for $u$ to be removed only if they are not currently inside a building. Here is a version that only modifies permission but has an additional guard, specifying that the user is not located in any building:

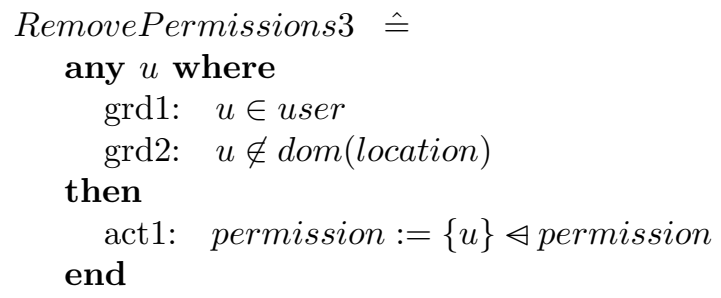

To see why this preserves the permission invariant we make use of the following rule; this states that if $x$ is not in the domain of relation $R$ then removing $x$ from the domain of $R$ has no effect:

\begin{tabular}{|c|c|}
\hline Description & Rule \\
\hline $\begin{array}{c}\text { Simplify } \\
\text { Domain Subtract }\end{array}$ & $x \notin \operatorname{dom}(R) \Rightarrow(\{x\} \triangleleft R)=R$ \\
\hline
\end{tabular}

The modified invariant resulting from RemovePermissions3 is

$$
\text { location } \subseteq \underline{\{u\} \triangleleft \text { permission }}
$$

Because of $\operatorname{grd} 1$ we can assume that $u \notin d o m$ (location) and therefore that location $=\{u\} \&$ location so this is the same as the following inclusion:

$$
\{u\} \triangleleft \text { location } \subseteq\{u\} \triangleleft \text { permission }
$$

As before, this inclusion follows from inv 5 by monotonicity.

We have seen that the RemovePermissions1 event does not preserve the permission inclusion invariant (inv5) while the other two versions of permission removal, RemovePermissions2 and RemovePermissions3, do preserve the invariant. Clearly we would want to rule out RemovePermissions1 since it fails to satisfy a mathematical judgement. Although RemovePermissions2 preserves the permission inclusion invariant, it does combine two separate functions into 
one atomic event. At the very least it would be more appropriate to reflect the dual role in the name the event, e.g., ExitAndRemovePermission. We note that location is a monitoring variable that is used to keep track of the physical location of users while permission is a conceptual variable that models a key concept in access control which does not reflect any physical entities. We prefer to keep changes to monitoring variables (e.g., location) separate from changes to conceptual variables (e.g., permission) so we use RemovePermissions3 as our specification of permission removal. This choice does mean that permission cannot be removed from a user for a building they are currently located in until after they exit the building. If it was deemed important, we might have a mechanism to force a user to exit a building but we treat this as out of scope of our analysis. Whenever the construction of the Event-B model raises ambiguities about the requirements (such as whether we can remove permissions for a user who is located in a building), then we should consider asking the system provider (the client) to clarify the requirements.

It is ok to remove permission from a user for a particular building even if they are located in another building? Here is an event that does this:

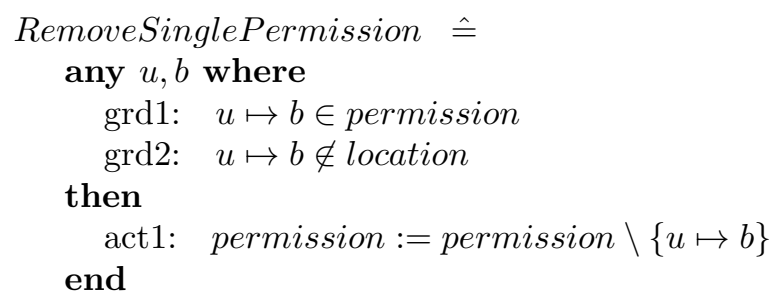

Here grd2 does not prevent $u$ from being located in some building $b^{\prime}$ that is different to $b$. To see why this event preserves inv5, consider the effect of the action act 1 on the invariant:

$$
\text { location } \subseteq \underline{\text { permission } \backslash\{u \mapsto b\}}
$$

Because of $\operatorname{grd} 2$, location $=$ location $\backslash\{u \mapsto b\}$, so this inclusion is the same as

$$
\text { location } \backslash\{u \mapsto b\} \subseteq \text { permission } \backslash\{u \mapsto b\}
$$

This inclusion follows from inv5 by monotonicity of set difference (set difference, set union and set intersection are all monotonic).

\subsection{Range subtraction}

The domain subtraction operator $(\varangle)$ is used to remove pairs from a relation based a domain set argument. There is also a range subtraction operator $(\triangleright)$ that removes pairs based on a range set argument. For example:

$$
\{u 1 \mapsto b 1, u 2 \mapsto b 3, u 4 \mapsto b 3\} \triangleright\{b 1\}=\{u 2 \mapsto b 3, u 4 \mapsto b 3\}
$$

Notice that in the case of domain subtraction, the set argument comes first and the relation comes second $(A \triangleleft R)$ while the arguments are swapped for the range operator $(R \triangleright B)$. The operator definition is as follows: 


\begin{tabular}{|c|c|c|}
\hline Name & Predicate & Definition \\
\hline $\begin{array}{c}\text { Range } \\
\text { Subtraction }\end{array}$ & $x \mapsto y \in R \triangleright B$ & $x \mapsto y \in R \wedge y \notin B$ \\
\hline
\end{tabular}

The event to remove a building from the registered buildings makes use of range subtraction to remove all permissions associated with that building:

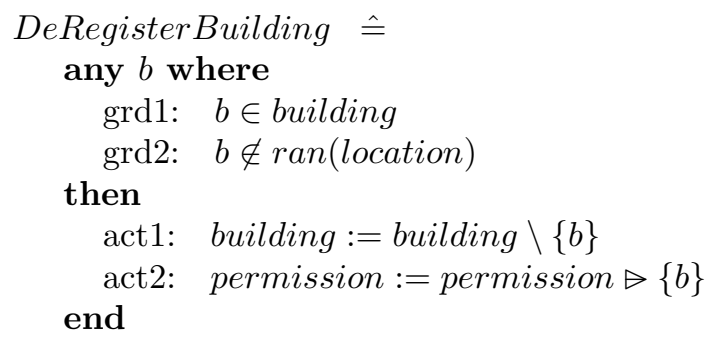

While $b$ is removed from building by action act 1 , action act 2 removes any permission associated with $b$ from permission. This is required in order preserve invariant inv3 which specifies that permission is a relation between user and building. The following rules show how the relational subtraction operators reduce the domain/range of a relation:

\begin{tabular}{|c|c|}
\hline Description & Rule \\
\hline Domain/Range & $R \in S \leftrightarrow T \Rightarrow$ \\
Reduction & $(A \triangleleft R) \in(S \backslash A) \leftrightarrow T$ \\
& $(R \triangleright B) \in S \leftrightarrow(T \backslash B)$ \\
& \\
\hline
\end{tabular}

Notice that grd2 of the DeRegisterBuilding event requires that $b$ has no occupants (no users are located in $b$ ). This ensures that the event maintains inv4 stating that the range of location is included in building and also maintains the permission inclusion invariant inv5 when the permissions for $b$ are removed by act2.

The event to de-register a user is specified as follows:

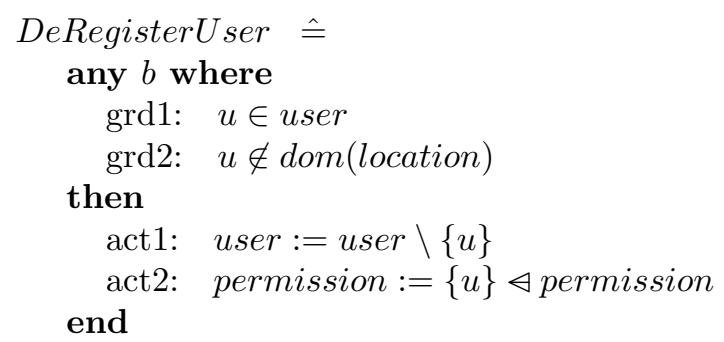

Guard $\operatorname{grd} 2$ requires that $u$ is not located in a building (preserving inv5), while action act 2 removes all permissions for $u$ (preserving $i n v 3$ ). 
For both of the de-register events, we required that the building is unoccupied (for DeRegisterBuilding) or the user is not in a building (for DeRegisterUser). This was to ensure the preservation of the invariant inv5. An alternative way of preserving inv5, that does not require guards on location, would be to reduce location as well, as we saw with Remove Permission2. As with permission removal, we prefer to keep location changes and user registration changes as separate events. This is again because location is a monitoring variable while the property of being registered is conceptual.

\section{Query events}

The events we have looked at so far all include actions that change one or more variables. Sometimes we are interested in querying information about a system such as the location of a user. Here is is the specification of such an event:

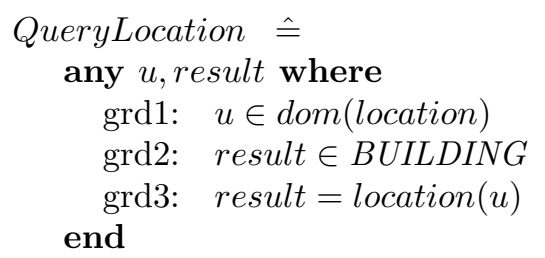

The event has two parameters: $u$, the user whose location is being queried, and result, the result of the query. In this case the result of the query is the location of $u$. The Event-B language does not have an explicit notion of an output parameter. We adopt the convention of naming a parameter representing an output as result. Typically the value of a result parameter will be defined by an exact equation such as grd3 above. We refer to an event that specifies a result but does not modify any variables as a query event.

The guard that specifies the type of the result in QueryLocation, grd2, is not strictly necessary since the type can be inferred from the equation in grd3. However making the type of result explicit makes the specification clearer.

Another query we could perform on the access control system would be to find out the set of buildings that a particular user has permission to enter. To do that we use the relational image operator. Given a relation $R \in S \leftrightarrow T$ and a set $A \subseteq S$, the expression $R[A]$ represents the set of range elements corresponding to some domain element in $A$. For example, consider again the following simple relation:

$$
\begin{aligned}
& \text { directory }=\{\text { mary } \mapsto 287573 \text {, } \\
& \text { mary } \mapsto 398620 \text {, } \\
& \text { john } \mapsto 829483 \text {, } \\
& \text { jim } \mapsto 398620\}
\end{aligned}
$$

If we want to identify the set of numbers that mary is mapped to, we write directory $[\{$ mary $\}]$ where

$$
\text { directory }[\{\text { mary }\}]=\{287573,398620\}
$$


Note the argument within the brackets must be a set of domain elements rather than a single element which is why we do not write directory [mary].

In general, a range element $y$ is in the relational image of $A$ under $R$ if there is some element $x$ in $A$ that is mapped to $y$ by $R$. This specified precisely in the following table:

\begin{tabular}{|c|c|c|}
\hline Name & Predicate & Definition \\
\hline Relational Image & $y \in R[A]$ & $\exists x \cdot x \in A \wedge x \mapsto y \in R$ \\
\hline
\end{tabular}

Suppose there are no elements of $A$ mapped to range elements by $R$. In that case there is no $x$ in $A$ satisfying $x \mapsto y \in R$ and therefore $R[A]$ will be empty.

The event to query the permissions of a user makes use of relational image:

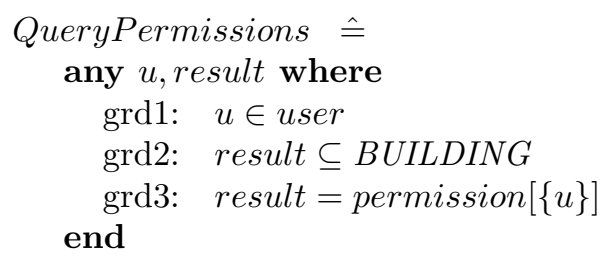

Here the result is specified as the relational image of $\{u\}$ under the permission relation, i.e., the set of buildings for which $u$ has permission. In the case that the user has no permissions, then the result will be the empty set.

We have seen that relational image allows us to specify a query on a relation going from domain elements to range elements. To perform a query in the opposite direction, from range to domain, we can take the inverse of a relation, written $R^{-1}$. The inverse of $R$ is the result of swapping the order of each pair in $R$. For example, the inverse of the directory relation specified above is as follows:

$$
\begin{aligned}
\text { directory }^{-1}=\{287573 & \mapsto \text { mary, } \\
398620 & \mapsto \text { mary } \\
829483 & \mapsto j o h n, \\
398620 & \mapsto j i m\}
\end{aligned}
$$

We can use this to query the people associated with phone number 398620 as follows:

$$
\text { directory }{ }^{-1}[\{398620\}]=\{\text { mary, jim }\}
$$

The inverse operator is defined in the following table:

\begin{tabular}{|c|c|c|}
\hline Name & Predicate & Definition \\
\hline Relational Inverse & $y \mapsto x \in R^{-1}$ & $x \mapsto y \in R$ \\
\hline
\end{tabular}


Using inverse and image, a query event that provides the set of users who have permission to enter building $b$ is specified as follows:

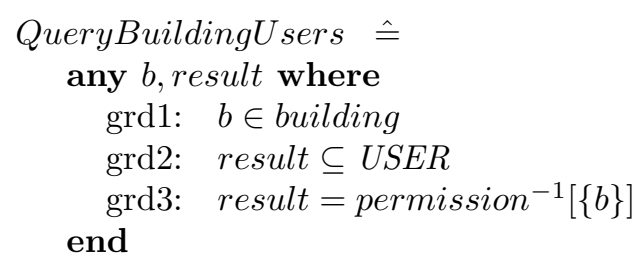

We can use a similar query event to provide the set of occupants of a building:

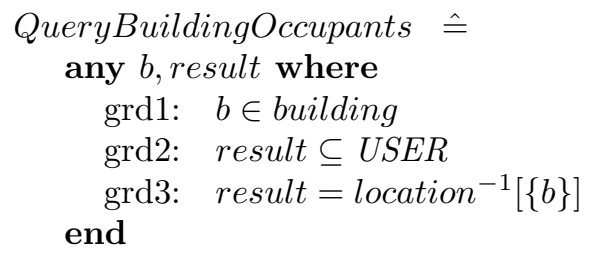

Note that while location is functional, the inverse of location might not be. This is illustrated by Figure 10 where two different users, $u 2$ and $u 4$ are located in $b 3$. This means that in the inverse relation, $b 3$ is mapped to two different users and thus location ${ }^{-1}$ is not functional. If a relation is one-to-one, e.g., Figure 12, then its inverse is also functional. A one-to-one function is also called injective and is declared as $f \in S \nrightarrow T$. An injective function is defined as a function whose inverse is also functional:

\begin{tabular}{|c|c|c|}
\hline Name & Predicate & Definition \\
\hline Injective Function & $f \in S \mapsto T$ & $f \in S \rightarrow T \wedge f^{-1} \in T \rightarrow S$ \\
\hline
\end{tabular}

\subsection{Requirements Tracing}

In order to be systematic about validation of the model against the requirements, we will re-visit the list of requirements and annotate each one with a explanation of how it is represented in the Event-B model. This is a form of tracing information: a means of tracing from a requirement through to a part, or parts, of the formal model. This is shown as a table in Figure 14 where the explanations of how a requirement is represented in the formal model are in shown in the second column. For example, the annotation on FUN1 provides an explanation of how that requirement is represented in the formal model through the user variable and the RegisterUser and UnRegisterUser events.

\section{Simple Bank}

We make use of some of the techniques shown so far to develop a model of a simple banking system. This case study emphasises the use of functions and 


\begin{tabular}{|l|l|}
\hline Requirement & Representation in model \\
\hline FUN1 & $\begin{array}{l}\text { This is represented by the user variable modelling registered } \\
\text { users (inv1) and by the RegisterUser and DeRegisterUser } \\
\text { events. } \\
\text { This is represented by the building variable modelling registered } \\
\text { buildings (inv2) and by the RegisterBuilding and DeRegister- } \\
\text { Building events. } \\
\text { This is represented by the permissions variable (inv3) and by } \\
\text { the AddPermission and RemovePermission events. }\end{array}$ \\
\hline FUN3 & $\begin{array}{l}\text { This is represented by the permission invariant (inv5) and by } \\
\text { the EnterBuilding event. Guard grd2 of the EnterBuilding event } \\
\text { ensures that the entering user has permission. }\end{array}$ \\
\hline FUN4 & $\begin{array}{l}\text { This is represented by the ExitBuilding event. There is no con- } \\
\text { straint on this event other than the user is located in a building } \\
\text { (grd1). }\end{array}$ \\
\hline FSM1 & $\begin{array}{l}\text { This is represented by the location being functional } \text { inv4). } \\
\text { This represented by grd1 of the EnterBuilding event which } \\
\text { building. }\end{array}$ \\
\hline ASM2 &
\end{tabular}

Fig. 14. List of requirement labels with tracing information

introduces an additional mathematical concept (function override). The case study also serves to re-enforce the steps that may be taken in developing an Event-B model from a list of requirements including the use of a class diagram to identify the main entities and the relationship between them. Here is a list of functional requirements for the simple bank:

- FUN1: The system shall maintain a register of bank customers and shall provide operations for managing the customer set.

- FUN2: The system shall maintain a name and address for each customer and shall provide operations for managing these.

- FUN3: The system shall allow customers to have several accounts and allow customers to share accounts.

- FUN4: The system shall provide operations for managing the account set.

- FUN5: The system shall maintain a balance for each account.

- FUN6: The system shall ensure that account balances are never negative.

- FUN7: The system shall provide operations for depositing and withdrawing funds to and from an account and for transferring funds between accounts.

\subsection{Sets and relations}

The first step in developing the model is to identify some carrier sets. In the functional requirements above we have highlighted some nouns in bold, e.g., customers in FUN1. Requirement FUN2 introduces names and addresses at a high level and does not define a specific format. We will treat names and 
addresses as abstract values and model them using carrier sets. These highlighted nouns suggest the carrier sets shown in Figure 15. Note that we only highlighted the first occurrence of a noun to avoid duplication. Figure 15 does not identify a carrier set for balance in FUN5. For simplicity we decide to model the amount of money in an account as an integer value; we could have chosen more detail such as currency units (e.g., euros) and subunits (e.g., cents). Integers are already part of the Event-B language.

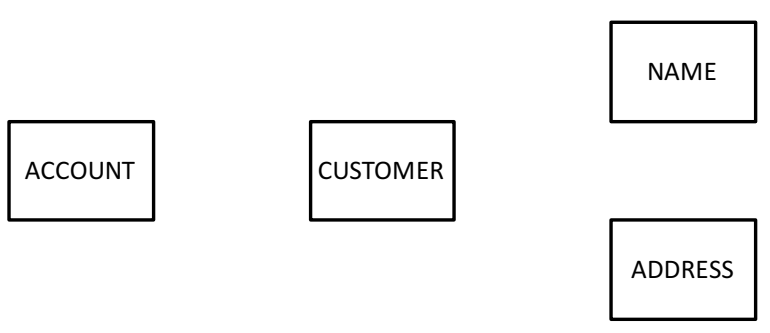

Fig. 15. Carrier sets for simple bank

Figure 15 gives rise to the following context for our simple bank model:

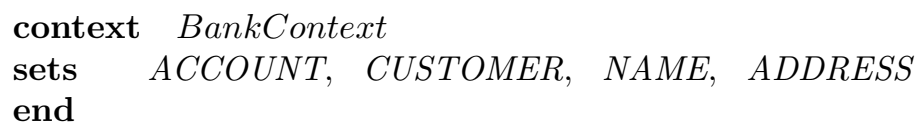

Next we identify whether any of the requirements suggest relations between sets. FUN2 suggests a relation between CUSTOMER and NAME and between CUSTOMER and ADDRESS. We name these relations name and address respectively and they are shown in Figure 16. FUN3 suggests a relation between CUSTOMER and ACCOUNT which we name as accounts. FUN5 suggests a relation between ACCOUNT and integers which we name as balance.

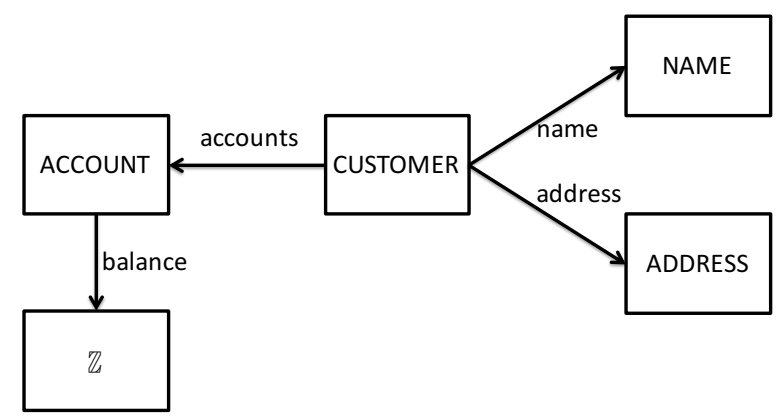

Fig. 16. Adding relations for the bank 
Having identified the main sets and required relationships between them, we add a bit more precision to the diagram. For the building access model we distinguished the (fixed) carrier sets for users and buildings from the (variable) registered sets and we do the same for the simple bank. In Figure 17 we have replaced the CUSTOMER and ACCOUNT carrier sets by the customer and account variable sets. The variable sets represent registered customers and accounts respectively and they allow us to represent constraints such as requiring customers to be registered in order to have accounts. In order to avoid confusion with the set account, we have changed the name of the relation between customer and account to cust_acc in Figure 17. The other way in which we finesse the diagram is to determine the nature of each relation (many-to-many, etc). Since FUN3 requires that a customers may have multiple accounts, we conclude that cust_acc should be a many-to-many relation. From FUN2 we conclude that each customer has one name and one address hence we conclude that these should be functional. We mark them as total functions from customer to indicate that each registered customer has a name and an address. Similarly from FUN5 we conclude that balance should be a total function from account.

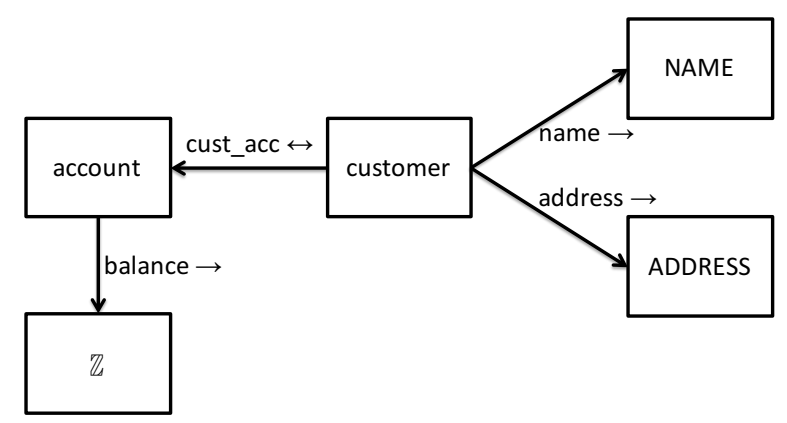

Fig. 17. Finessing the bank model

We have not introduced variable sets corresponding to NAME nor ADDRESS. The reason is that we regard these sets as secondary. By this we mean that we are not interested in the values from these sets in their own right and we are only interested in them as attributes of the other sets. We refer to the non-secondary sets (customer, account) as primary. One indicator of a secondary set is that it has no outgoing arrows in the class diagram, and only has incoming arrows. This is the case for the sets NAME, ADDRESS and indeed $\mathbb{Z}$ in Figure 17. However this is not a hard-and-fast rule: the building set of Figure 13 has no out-going arrows but we still treat it as a primary set since the requirements explicitly stated that a set of registered buildings should be maintained. In the simple bank there is no requirement to maintain a set of registered addresses and names independent of the customer to which they belong. Neither is there 
a requirement to maintain a set of balance values independent of the accounts to which they belong.

Construction of the class diagram of Figure 17 allows to identify the set and relation variables. The primary sets customer and account become variable sets while the relations name, address, cust_acc andbalance become relation variables:

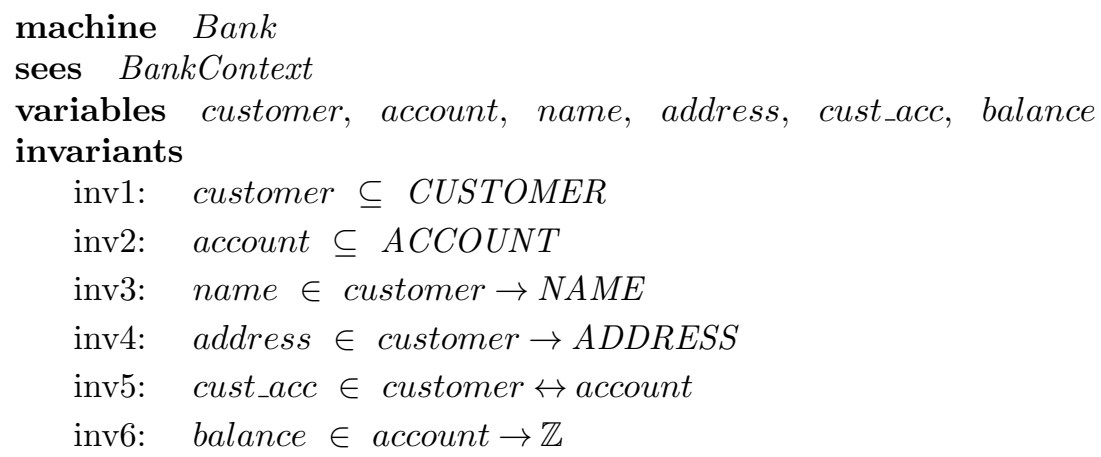

An advantage of having the variable set customer in the model is that it allows us to specify that the functions name and address have exactly the same domain. All of the above invariants are derived directly from Figure 17 (which in turn was derived from the requirements via the other two class diagrams).

We study the requirements again to check if there are any further invariants we could identify. The only requirement from which we can identify a further invariant is FUN6 which states that account balances are never negative (a rather conservative requirement for a bank!). We can represent this requirement by strengthening inv6 to specify that the range of balance is the set of naturals rather than integers (naturals are written $\mathbb{N}$ and represent all the non-negative integers, i.e., those $n \in \mathbb{Z}$ where $n \geq 0)$ :

\section{invariants \\ ... inv6b: $\quad$ balance $\in$ account $\rightarrow \mathbb{N}$}

An alternative formulation of FUN5 is to specify that the balance of each account is non-negative using universal quantification:

\section{invariants}

$\cdots$

inv6: balance $\in$ account $\rightarrow \mathbb{Z}$

inv7: $\forall a \cdot a \in$ account $\Rightarrow$ balance $(a) \geq 0$

In $i n v 7$ we restrict the quantification to those $a$ in the set account. Since balance is total on the set account, the expression balance $(a)$ is guaranteed to be welldefined.

We are not yet done with identifying invariants. Although we might not be able to identify this explicitly from the requirements, we need to be careful about the domain and range of the cust_acc relation. Invariant inv5 specifies that the 
domain of cust_acc is a subset of customer but does not specify that the domain is equal to customer. This means we may have customers who have no accounts associated with them. Similarly inv 5 allows for register accounts that have no customers associated with them. The requirements are not clear on this and we now have the opportunity to be more precise.

We decide that we may have a customer who has no accounts, e.g, this might arise when we register a customer before we create any accounts for them. Thus dom(cust_acc) does not need to equal customer and can be a subset. However, we decide that it is not ok to have an account that has no customers associated with it. We introduce a further invariant to specify that every account is associated with some customer:

\section{invariants}

$\cdots$

inv8: $\quad$ ran $($ cust_acc $)=$ account

The combination of inv5 and inv8 means that each customer has zero or more accounts, while each account has one or more customers.

\subsection{Expansion events}

We introduced a distinction between primary and secondary sets and we identified that customer and account as the primary sets in Figure 17. It is for the primary sets that we introduce expansion events (events that expand some set of elements). The customer set is expanded by the event for registering a new customer:

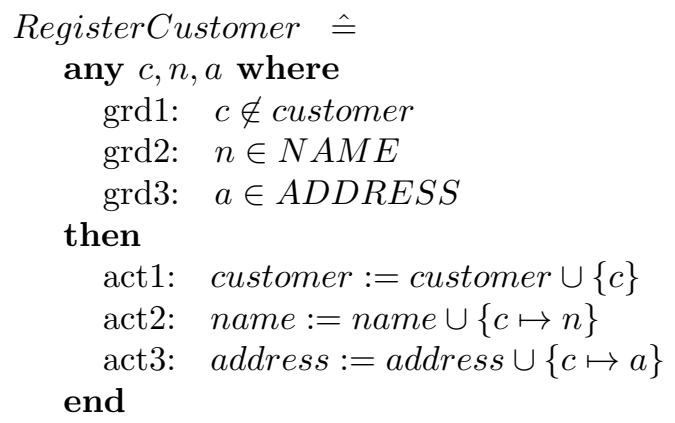

Similar to registration of new users in the building access example, the new customer $c$ is represented by a 'fresh' value ( $\operatorname{rrd} 1)$. Since we are expanding customer (act1), and since name and address are total functions on customer, we also need to expand name and address (act2 and act3). The values for the name and address of the new customer are provided as parameters $n$ and $a$.

The following rule shows how extending a total function maintains functionality. It shows that the extended function $(f \cup\{x \mapsto y\})$ is total on an expanded domain $(S \cup\{x\})$. 


\begin{tabular}{|c|c|}
\hline Description & \multicolumn{1}{|c|}{ Rule } \\
\hline Total & $f \in S \rightarrow T \wedge$ \\
Function & $x \notin S \wedge y \in T$ \\
Extension & $\Rightarrow(f \cup\{x \mapsto y\}) \in(S \cup\{x\}) \rightarrow T$ \\
&
\end{tabular}

Our RegisterCustomer event does not associate any accounts to the new customer. This does not violate any invariants since we concluded that a customer may have zero or more accounts. Since we also concluded that an account must have at least one associated customer (inv8), we need to associate at least one customer with a newly created account. We chose to associate a set of customers with a newly created account and this set will need to be non-empty. Since balance is total on account, we also need to associate a balance value with the newly created account; we will set the balance to be zero. We specify the event as follows:

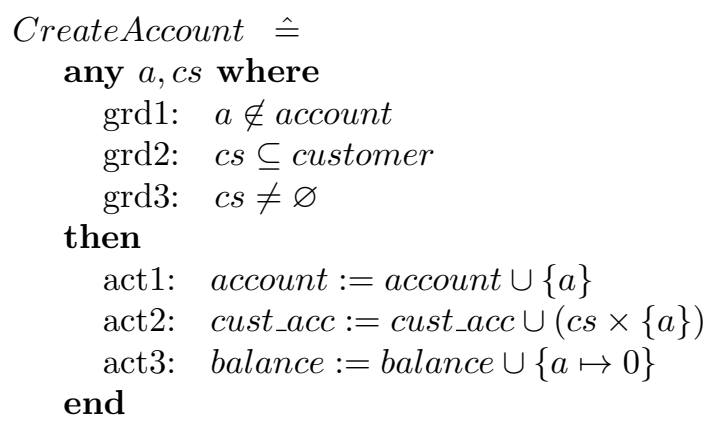

A note on the naming of these events: we used 'register' to name expansion event for customers (RegisterCustomer) while we used 'create' for accounts (CreateAccount). The reason is that values in customer correspond to entities that are external to the bank while accounts are entities that are internal to the bank. To use our previously-introduced terminology, customer is a monitoring variable while account is a conceptual variable. Of course naming is matter of taste and judgement. Our distinction between registration and creation is simply a guideline.

The above expansion events contribute to addressing the requirements for 'managing' the set of accounts (FUN1) and the set of accounts (FUN4). Both these requirements also suggest reduction events for the primary sets, e.g., DeRegisterCustomer and DeleteAccount. FUN4 also suggests events for expanding and reducing the set of customers associated with an account, e.g., AddAccountCustomer and RemoveAccountCustomer. We leave the specification of these to the reader. As before, care must be taken to ensure that all the invariants are preserved by these reduction events. 


\subsection{Function override}

Requirement FUN2 suggests events for modifying the address of a customer and possibly even the name of a customer. FUN7 suggests events for increasing and decreasing the balance of an account and for transferring funds between accounts. Specification of all of these involve modifying a function so that the range value that some domain element is mapped to is updated, e.g., to withdraw money from account $a$, the balance function gets updated so that the value associated with $a$ is changed to a smaller value. To represent function update mathematically we use the function override operator.

We illustrate the use of this operator with an example first. Assume that the balance function has the following value:

$$
\text { balance }=\{a 1 \mapsto 100, a 2 \mapsto 350, a 3 \mapsto 800, a 4 \mapsto 50\}
$$

If we want to change the balance of account $a 2$ to 300 , we use function override $(\varangle)$ with balance as the first argument and a mapping from $a 2$ to 300 as the second argument, written balance $\varangle\{a 2 \mapsto 300\}$. The following equation shows the result of this overriding:

$$
\text { balance } \varangle\{a 2 \mapsto 300\}=\{a 1 \mapsto 100, a 2 \mapsto \underline{300}, a 3 \mapsto 800, a 4 \mapsto 50\}
$$

As highlighted in the resulting function on the right-hand side, 350 has been replaced by 300 . Function override is a combination of domain subtraction and set union, i.e., $f \nLeftarrow\{a \mapsto b\}$ is the same as removing the existing mapping for $a$ from $f$ using domain subtraction and adding the updated mapping using union:

$$
f \nLeftarrow\{a \mapsto b\}=(\{a\} \triangleleft f) \cup\{a \mapsto b\}
$$

More generally, the second argument for function override is itself a function, $f \nLeftarrow g$, rather than just a singleton mapping $f \nLeftarrow\{a \mapsto b\}$. The general definition also uses domain subtraction and set union as shown in the following table:

\begin{tabular}{|c|c|c|}
\hline Name & Expression & Definition \\
\hline Function override & $f \leftrightarrow g$ & $(\operatorname{dom}(g) \triangleleft f) \cup g$ \\
\hline
\end{tabular}

The specification of the event for depositing money in an account, IncreaseBalance, uses function override to update the value of balance:

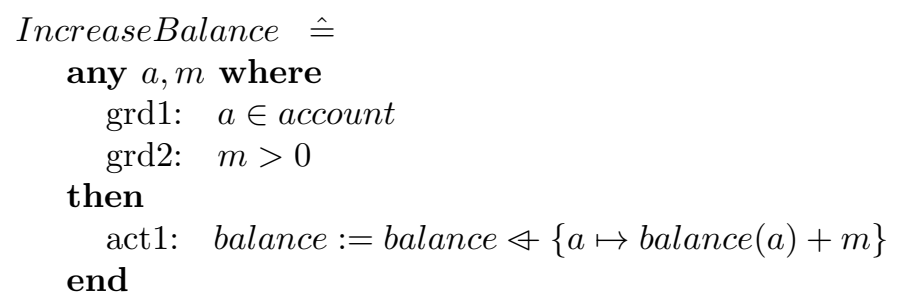


Here $m$ is the amount to be deposited in account $a$. We require $m$ to be greater than zero since adding zero would seem rather pointless (grd2). In act 1 the balance of account $a$ is updated to the value balance $(a)+m$.

It is worth noting the difference between extending a function using union and updating a function using function override. Function extension is used when adding a new value to the domain, e.g., expanding the domain of balance when creating an account. Function override is used when modifying the range value associated with an existing domain element, e.g., modifying the balance of an existing account when depositing money.

The following rules about function override support mathematical reasoning. The first rule shows the conditions under which a function override $(f \nLeftarrow\{x \mapsto y\})$ remains a total function. The second rule shows that the result of applying a function override $(f \&\{x \mapsto y\})$ to domain value $w$ depends on whether $w$ is the same as or different to $x$ :

\begin{tabular}{|c|c|}
\hline Description & Rule \\
\hline Total & $f \in S \rightarrow T \wedge$ \\
Function & $x \in S \wedge y \in T$ \\
Update & $\Rightarrow(f \triangleleft\{x \mapsto y\}) \in S \rightarrow T$ \\
& \\
\hline Apply & $f \in S \rightarrow T \wedge w \in S \Rightarrow$ \\
Function & $w=x \Rightarrow(f \&\{x \mapsto y\})(w)=y$ \\
Update & $w \neq x \Rightarrow(f \&\{x \mapsto y\})(w)=f(w)$ \\
& \\
\hline
\end{tabular}

The shape of action act 1 in IncreaseBalance is a common one when updating functions at a single domain point, i.e., it has the form $f:=f \&\{x \mapsto E\}$. Because update of a function at a single point is a common action in Event-B, it may be written in a simple syntactic form $f(x):=E$. This syntactic form is defined by the following table:

\begin{tabular}{|c|c|c|}
\hline Name & Action & Definition \\
\hline Function single assignment & $f(x):=E$ & $f:=f \nLeftarrow\{x \mapsto E\}$ \\
\hline
\end{tabular}

Using this form, the IncreaseBalance event is specified as follows:

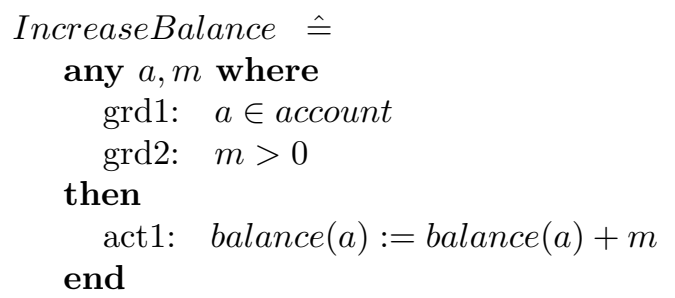


The DecreaseBalance event is specified in a similar way with the balance being decreased:

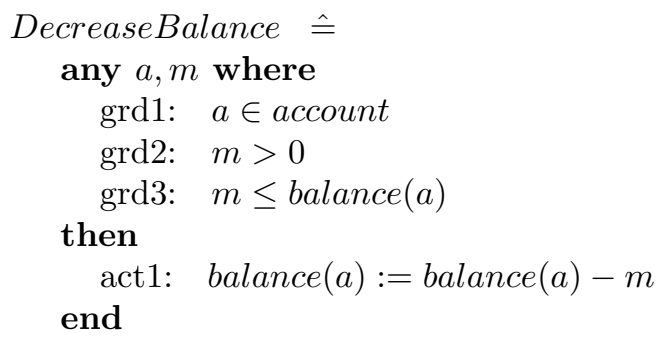

With this event, the amount to be withdrawn should not exceed the current balance of the account $(g r d 3)$. This is to ensure that the balance does not go negative (inv7). Let us reason about this more precisely. Recall that inv7 is a quantification over accounts as follows:

$$
\text { inv7: } \forall a \cdot a \in \text { account } \Rightarrow \text { balance }(a) \geq 0
$$

Action act 1 of DecreaseBalance is equivalent to assigning an overridden function to balance and so gives rise to the following modified invariant:

$$
\forall a^{\prime} \cdot a^{\prime} \in \text { account } \Rightarrow \underline{(\text { balance } \varangle\{a \mapsto \text { balance }(a)-m\})}\left(a^{\prime}\right) \geq 0
$$

Note that here we have renamed the quantified variable $a$ to $a^{\prime}$. This is to avoid a name clash with the event parameter $a$. The Apply Function Update rule shown above suggests that we reason about (7) by considering two cases: $a=a^{\prime}$ and $a \neq a^{\prime}$.

In the case that $a=a^{\prime},(7)$ is simplified by the Apply Function Update rule to the following:

$$
\forall a^{\prime} \cdot a^{\prime} \in \operatorname{account} \wedge a^{\prime}=a \Rightarrow \operatorname{balance}(a)-m \geq 0
$$

This is equivalent to balance $(a) \geq m$ which follows from $\operatorname{grd} 3$ of DecreaseBalance.

In the case that $a \neq a^{\prime},(7)$ is simplified to the following:

$$
\forall a^{\prime} \cdot a^{\prime} \in \text { account } \wedge a^{\prime} \neq a \Rightarrow \operatorname{balance}\left(a^{\prime}\right) \geq 0
$$

This follows from inv7.

Requirement FUN7 requires an event for transferring money from one account, $a$, to another account, $b$. This is specified as follows, and as with the DecreaseBalance event, requires that the amount to be transferred does not exceed the balance of the source account $a$ : 


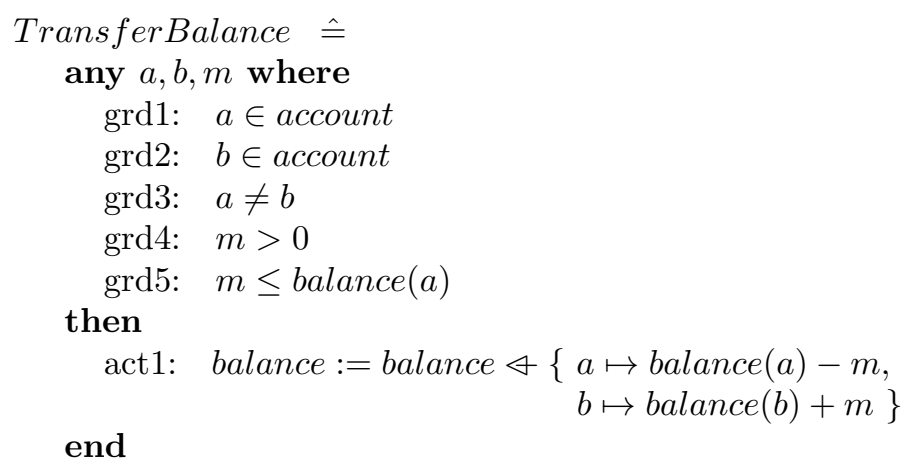

Note that grd3 requires that the source and target accounts are distinct (to avoid pointless transfers). The action act 1 uses function override to update the two account balances simultaneously. We might be tempted to write the actions of TransferBalance as two single updates as follows:

act1: $\quad$ balance $(a):=$ balance $(a)-m$

act2: balance $(b):=$ balance $(b)+m$

This is syntactically invalid in Event-B as it involves two actions assigning to the same variable in a single event and so we avoid this form.

We can introduce event parameters representing values local to the event to increase the readability of the specification of TransferBalance:

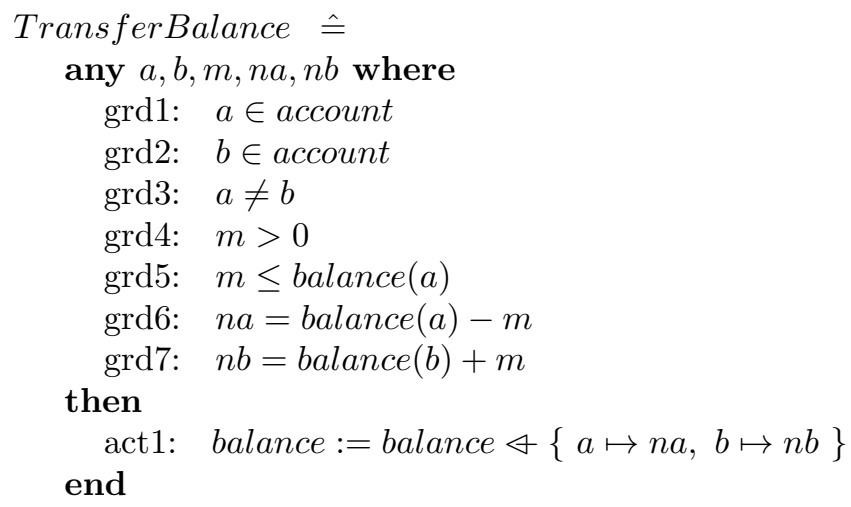

Here $n a$ and $n b$ represent the new balances of $a$ and $b$ respectively whose values are defined by $\operatorname{grd} 6$ and $\operatorname{grd} d$.

Events to update the name or address of a customer can also be specified using function override. We leave these to the reader. The requirements do not explicitly mention queries on the bank data such as the balance of an account or the customers associated with an account. We leave these for the reader to specify. 


\section{Model validation through animation}

A very useful validation technique for Event-B models is to use an animation tool such as ProB [4] or AnimB ${ }^{2}$. With these tools, the carrier sets are instantiated with some illustrative values, e.g., the carrier set USER is instantiated with the values $u 1, u 2, u 3$, and the model can be executed with these values. The execution is driven by the modeller and at each step the state can be inspected. For the purposes of animating our access control model, let us assume that the carrier set USER is instantiated with the values $u 1, u 2, u 3$ and that BUILDING is instantiated with the values $b 1, b 2, b 3$. Figure 18 represents the state that is reached by executing the following sequence of events on our model of the building access control system:

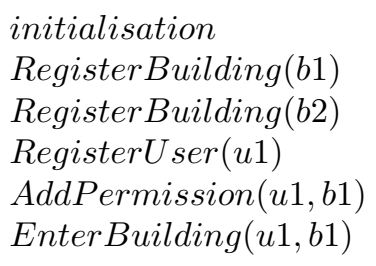

Figure 18 shows the values of the sets user and building and the relations permission and location as tables. The user and building tables show that there is one registered user and two registered buildings. The permission table shows that $u 1$ has permission to enter $b 1$ while the location table shows that $u 1$ is located in building $b 1$. These values for the variables are what we would expect to see after execution of the given sequence of events. Figure 18 also shows the events that are enabled in the reached state. We see that two more users $(u 2, u 3)$ and one more building (b3) can be registered. At the bottom of the list of enabled events we see that user $u 1$ may leave building $b 1$. We can see that the EnterBuilding event is not in the list of enabled events. This is as expected since the only registered user, $u 1$, is currently in building $b 1$ and there is no means to directly enter one building from another.

The value of the animation is that it helps us make human judgements about whether the behaviour specified by the model is what we would expect given the informal requirements. In this case we can make a judgement that the values of the tables correspond to what we would expect after the given sequence of event executions is performed. Inspecting the enabled events allows to check that the guards of the events are sufficiently strong, e.g., the fact that EnterBuilding is not in the list of enabled events in Figure 18 helps us to validate the guards specified for that event.

The event sequence above registers two buildings and one user. Here is a second event sequence that continues from the first, adding a second user $u 2$,

\footnotetext{
$\overline{2}$ www.animb.org
} 


\begin{tabular}{|c|c|c|c|c|}
\hline$u 1$ & $b 1, b 2$ & $u 1$ & $b 1$ & $u 1$ \\
\hline user & building & per & ssion & location \\
\hline & & d Ev & & \\
\hline & $U \operatorname{ser}(u 2)$ & terl & $\operatorname{er}(u 3)$ & \\
\hline & ter User ( & & & \\
\hline & Building & & & \\
\hline & terBuildi &,$D e$ & egiste & ing $(b 2)$ \\
\hline & ission $(u$ & & & \\
\hline$R \epsilon$ & Permissi & & & \\
\hline$E$ & $\operatorname{ding}(u 1)$ & & & \\
\hline
\end{tabular}

Fig. 18. Result of animating model through first sequence of events

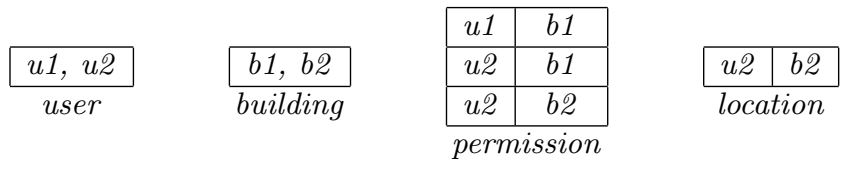

\begin{tabular}{|l|}
\hline \multicolumn{1}{|c|}{ Enabled Events } \\
\hline RegisterUser $(u 3)$ \\
\hline DeRegisterUser $(u 1)$, DeRegisterUser $(u 2)$ \\
\hline RegisterBuilding $(b 3)$ \\
\hline DeRegisterBuilding $(b 1)$, DeRegisterBuilding $(b 2)$ \\
\hline AddPermission $(u 1, b 2)$ \\
\hline RemovePermissions $(u 1)$, RemovePermissions $(u 2)$ \\
\hline EnterBuilding $(u 1, b 1)$ \\
\hline ExitBuilding $(u 2)$ \\
\hline
\end{tabular}

Fig. 19. Result of animating model through second sequence of events

giving that user permissions, entering $u 2$ in building $b 2$ and exiting user $u 1$ :

$$
\begin{aligned}
& \text { RegisterUser }(u 2) \\
& \text { AddPermission }(u 2, b 1) \\
& \text { AddPermission }(u 2, b 2) \\
& \text { EnterBuilding }(u 2, b 2) \\
& \text { ExitBuilding }(u 1)
\end{aligned}
$$

The state resulting from continuing from the state of Figure 18 is shown in Figure 19. In this figure, $u 2$ has been added to user, two rows have been added to permission and the location table has been updated. We see that an animation tool allows us to execute sequences of events on sample data values and inspect the effect of these on a representation of the state of a machine and on the enabledness of events. 


\begin{tabular}{|c|c|c|c|c|c|}
\hline$u 1, u 2$ & $b 1, b 2$ \\
\hline user & building & permission & & location \\
\hline
\end{tabular}

Fig. 20. Incorrect state reached when RemovePermissions1(u2) is applied to state in Figure 19

\section{Model verification}

Manual inspection of the tables in Figures 18 and 19 shows that they both represent states satisfying invariants inv 1 to inv5. However, rather than using manual inspection to check for satisfaction of invariants, model verification can be used to do this in a systematic and automated way. Model verification involves making mathematical judgements about the model. The main mathematical judgement we apply to the abstract model is to determine whether the invariants are guaranteed to be maintained by the events. Mathematical judgements are formulated as proof obligations (PO). These are mathematical theorems whose proof we attempt to discharge using a deductive proof system. In the Rodin toolset [5] for Event-B, mechanical proof of POs may be complemented by the use of the ProB model checker which searches for invariant violations by exploring the reachable states of a model.

Previously we argued that the RemovePermissions1 event could violate the permission inclusion invariant (inv5). Let us see how this plays out in animation of the model. Consider the state of the access control system shown in Figure 19. As already explained, this state is reachable by executing a particular sequence of events. In this state, $u 2$ is in building $b 2$ and has permission to be there. Now if the next event to be performed was RemovePermissions1(u2), the state reached would be as shown in Figure 20. This new state is an incorrect state, that is, it violates the permission inclusion invariant since user $u 2$ is still in building $b 2$ even though $u 2$ not longer has permission to be there. Indeed, ProB can automatically find a sequence of events that lead to an invariant violation (known as a counterexample). The counterexample that leads to the state in Figure 20 is not the shortest possible counterexample. ProB can automatically find a shorter counterexample that leads to violation of the permission inclusion invariant such as the following:

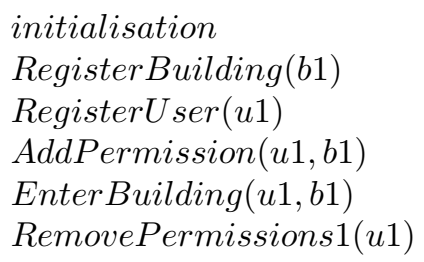

We look at how the error is reflected in the proof obligation (PO) for invariant preservation. Figure 21 shows a definition of this PO. The left side of the figure provides a schematic specification of an event $E$ with a guard represented 


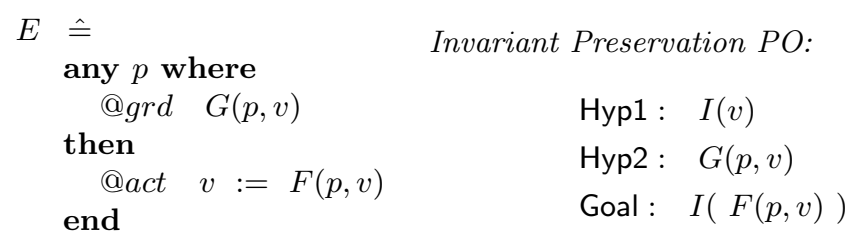

Fig. 21. Invariant preservation proof obligation for an event

by $G(p, v)$ and an action represented by $F(p, v)$. Here $p$ represents the event parameters and $v$ represents the variables on the machine on which the event operates. We write $G(p, v)$ to indicate that $p$ and $v$ are free variables of the predicate $G$. Assuming that $I(v)$ represents a invariant of the machine, the right hand side of Figure 21 shows the PO used to prove that the invariant is maintained by event $E$. The PO is in the form of a list of hypotheses and a goal. The PO is discharged by proving that the goal is true assuming that the hypotheses are true. In this case, the hypotheses are the invariant itself (Hyp1) and the guard of the event (Hyp2). The goal is the invariant with the free occurrences of variable $v$ replaced by $F(p, v)$, the value assigned to $v$ by the action of the event.

The Rodin tool for Event-B generates the invariant preservation POs for all of the events of the access control model and the automated provers of Rodin are able to discharge all of the generated POs except for one: the specification of the RemovePermissions1 event together with invariant inv5 give rise to the following PO that cannot be proved:

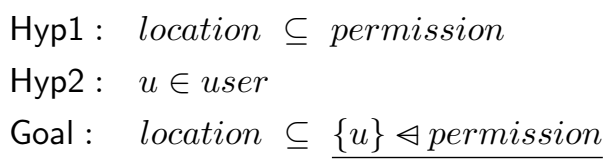

Here, Hyp1 is the invariant to be preserved and Hyp2 is the guard of the event. The event makes an assignment to the permission variable and thus the goal is formed by substituting permission by $\{u\} \triangleleft$ permission. The result of the substitution is underlined in the goal. The problem here is that the right-hand side of the set inequality in the goal, $\{u\} \triangleleft$ permission, is reduced compared with that in the hypothesis, Hyp1, while the left-had side, location, remains unchanged (as discussed in Section 8.4).

To address this problem with the RemovePermissions1 event, we provided two alternative specifications of permission removal. For example, the specification of the RemovePermissions3 event together with inv5 gives rise to the following PO that can be proved beause of the additional hypothesis provided by the additional guard:

$$
\begin{array}{ll}
\text { Hyp1: } & \text { location } \subseteq \text { permission } \\
\text { Hyp2a : } & u \in \text { user } \\
\text { Hyp2b : } & u \notin \text { dom(location }) \\
\text { Goal : } & \text { location } \subseteq\{u\} \triangleleft \text { permission }
\end{array}
$$


The counterexample generated by the ProB model checker highlighted a problem with the specification of the RemovePermissions1 event. This stronger condition for removing permission was identified through our attempt to prove that the original specification of the event maintained the permission inclusion invariant, leading to RemovePermission3. It is appropriate that we make a (human) judgement about the validity of this stronger specification of removing authorisation. Is it a reasonable constraint? Well, if we expect the access control policy to hold always, we have no choice: without the stronger guard, the event cannot maintain the permission inclusion invariant. We could remove the invariant completely from the model but that seems like an unsatisfactory solution since it would mean we were not addressing the main purpose of access control in our formalisation and would undermine what we can reasonably state in our requirements. For the purposes of this paper, we make the judgement that the invariant should stay and thus the revised version of the event, RemovePermission3, with the stronger guards holds.

\section{Further Reading}

Refinement is a key concept in Event-B and is used for structuring complex specifications and for relating abstract models with more concrete, implementationoriented models. We have not covered refinement in this paper because of space limitations. For a comprehensive introduction to modelling, refinement and proof in Event-B see Abrial's book on the topic [1]. For an overview of the role and practice of refinement in Event-B see [6]. Event-B evolved from the B Method which was also developed by Abrial [7]. The B Method was developed to model and reason about software systems and has module structuring mechanisms similar to modular programming languages. Event-B was designed to reason about systems that may include hardware and physical components as well as software. Some component-based structuring mechanisms for Event-B are described in [8].

In Section 4.6 we saw that the choice of value for a parameter is treated as nondeterministic: any value that satisfies the guards may be chosen. In Event-B, it is also possible to specify nondeterministic actions of the following form [1]:

$$
v:=v^{\prime} \mid P\left(v, v^{\prime}\right)
$$

Here $P\left(v, v^{\prime}\right)$ is a predicate that describes a relation between the before and after values of variable $v$. The nondeterministic action states that $v$ should be assigned a new value $v^{\prime}$ such that $P\left(v, v^{\prime}\right)$ holds. For example, assuming that $x$ is an integer variable, then the following action increases $x$ by a nondeterministic amount:

$$
x:=x^{\prime} \mid x<x^{\prime}
$$

Nondeterministic actions have a feasibility proof obligation which requires that there exists some value $v^{\prime}$ satisfying $P\left(v, v^{\prime}\right)$ when the invariant and event guards hold [1]. In this paper, we only made use of deterministic actions and used the choice of parameter values to represent nondeterminism within an event. 
Our reason for using this style is that it allows the nondeterministically chosen value to be available across all of the actions of an event. For example, in the RegisterUser2 event in Section 4.5, the parameter $u$ is used in both actions so that the same nondeterministically chosen value for $u$ is added to register and to out.

The mathematical language of Event-B (logic and set theory) is similar to the mathematical language of the B Method. These in turn were influenced by the $\mathrm{Z}$ notation [9] and VDM [10]. The use of class diagrams to aid the construction of Event-B models, as used in this paper, was inspired by the UML-B notation which provides a graphical syntax for parts of Event-B [11].

For more information on the Rodin tool see [5]. The Rodin tool can be downloaded via the Event-B.org ${ }^{3}$ website which also contains a Rodin User Manual ${ }^{4}$. The Atelier B tool ${ }^{5}$ supports the B Method. For details of the ProB tool see [4] and the ProB website ${ }^{6}$. ProB is available as a plug-in for Rodin as is the AnimB tool $^{7}$.

\section{Concluding}

This paper provided an overview of how the Event-B language and verification method can be used to model and reason about system behaviour. Reasoning about the system is not just about proving invariant properties. Several different forms of reasoning were deployed in addition to mathematical reasoning: identification of the main purpose of a system, abstraction from design details in requirements, identification of the various entities in the system and their relationships - all of these are forms of reasoning. Constructing the formal model based on the requirements is another form of reasoning as is validation of the model against the requirements through human judgement. All these forms of reasoning complement each other in helping us to understand the purpose of a system and the constraints on the system.

Event-B encourages us to identify the main entities of the problem under consideration and the relationships between those entities. It also encourages us to identify what properties should always hold (invariants), under what conditions system transitions are allowed (guards) and the effect of those transitions on the system state (actions). We have seen how the mathematical structures chosen can encourage us to identify different kinds of events such as set expansion events, set reduction events and query events.

This paper emphasised mathematical reasoning as this is a particular strength of a specification language such as Event-B. The paper presented definitions and rules in order to help the reader gain a strong understanding of the mathematical

\footnotetext{
3 www.event-b.org

4 www3.hhu.de/stups/handbook/rodin/current/html/index.html

${ }^{5}$ www.atelierb.eu/en/outil-atelier-b/

${ }^{6}$ www3.hhu.de/stups/prob/index.php/The_ProB_Animator_and_Model_Checker

7 www.animb.org
} 
operators and their properties. Understanding the properties of the mathematical operators helps ensure that we are choosing the appropriate operators in order to specify an intended effect. It allows us to check that the mathematics is being used in an appropriate way, both from a validation point of view (is the specification meeting the requirements?) and a correctness point of view (is the specification maintaining invariants?).

Many of the invariants used in this paper were in the form of equations $(E=F)$ and inclusions $(E \subseteq F)$. Typically the actions of an event modify one or both sides of an equation or inclusion. We used two main ways of preserving the equations and inclusions: either adding sufficient actions to ensure both sides of an equation or inclusion are modified in similar ways or using guards and properties of the operators to verify that modifying only one side still preserves the equation or inclusion.

We quoted Boehm's First Law in the introduction. Let us quote Boehm's Second Law [2] in the conclusion:

Boehm's Second Law: Prototyping significantly reduces requirements and design errors, especially for user errors.

We would argue that a formal model in a language such as Event-B acts as a form of early prototype allowing us to uncover and fix errors in requirements. Of course, while formal modelling addresses the key concepts in the problem being solved by a software system, it does not deal with the important issue of user interfaces (which can cause the user errors referred to in Boehm's Second Law); a software prototype remains an important tool in uncovering requirements on user interfaces. Formal modelling and reasoning help to uncover conceptual errors in requirements while software prototypes help uncover user interface errors.

We conclude by summarising some key messages:

- The role of problem abstraction and formal modelling is to increase understanding of a problem leading to good quality requirements and design documents with low error rates.

- The role of model validation is to ensure that formal models adequately represent the intended behaviour of a system.

- The role of model verification is to improve the quality of models through formulation of invariants and reasoning about those invariants, including rectifying specifications where appropriate.

\section{References}

1. Abrial, J.R.: Modeling in Event-B: System and Software Engineering. Cambridge University Press (2010)

2. Boehm, B.W.: Software Engineering Economics. 1st edn. Prentice Hall PTR, Upper Saddle River, NJ, USA (1981)

3. Feiler, P., Goodenough, J., Gurfinkel, A., Weinstock, C., Wrage, L.: Four pillars for improving the quality of safety-critical software-reliant systems. Technical report, Software Engineering Institute, Carnegie-Mellon University (2013) https: //resources.sei.cmu.edu/asset_files/WhitePaper/2013_019_001_47803.pdf. 
4. Leuschel, M., Butler, M.: ProB: An automated analysis toolset for the B Method. Intl. J. on Software Tools for Technology Transfer 10(2) (2008) 185-203 http: //eprints.soton.ac.uk/262886/.

5. Abrial, J.R., Butler, M., Hallerstede, S., Hoang, T., Mehta, F., Voisin, L.: Rodin: an open toolset for modelling and reasoning in Event-B. STTT 12(6) (2010) 447-466 http://dx.doi.org/10.1007/s10009-010-0145-y.

6. Butler, M.: Mastering system analysis and design through abstraction and refinement. In: Engineering Dependable Software Systems, IOS Press (2013) http: //eprints.soton.ac.uk/349769/.

7. Abrial, J.R.: Modeling in Event-B: System and Software Engineering. Cambridge University Press (1996)

8. Silva, R., Pascal, C., Hoang, T., Butler, M.: Decomposition tool for Event-B. Software: Practice and Experience 41(2) (February 2011) 199-208 http://www. eprints.soton.ac.uk/271714/.

9. Woodcock, J., Davies, J.: Using Z - Specification, Refinement, and Proof. PrenticeHall (1996) http://www. usingz.com.

10. Jones, C.: Systematic Software Development using VDM. Prentice Hall (1990)

11. Snook, C., Butler, M.: UML-B: Formal modelling and design aided by UML. ACM Transactions on Software Engineering and Methodology 15(1) (January 2006) 92122 http://eprints. soton.ac.uk/260169/. 